\title{
Robust Event-Triggered Energy-to-Peak Filtering for Polytopic Uncertain Systems over Lossy Network with Quantized Measurements
}

\author{
Jidong Wang ${ }^{1}$ and Kezhen Han ${ }^{2}$ \\ ${ }^{1}$ School of Electrical Engineering, North China University of Water Resources and Electric Power, Zhengzhou 450011, China \\ ${ }^{2}$ School of Information Science and Engineering, Northeastern University, Shenyang 110819, China \\ Correspondence should be addressed to Jidong Wang; kfwjd@ncwu.edu.cn
}

Received 13 December 2015; Revised 20 February 2016; Accepted 29 March 2016

Academic Editor: Filippo Cacace

Copyright (c $2016 \mathrm{~J}$. Wang and K. Han. This is an open access article distributed under the Creative Commons Attribution License, which permits unrestricted use, distribution, and reproduction in any medium, provided the original work is properly cited.

\begin{abstract}
The event-triggered energy-to-peak filtering for polytopic discrete-time linear systems is studied with the consideration of lossy network and quantization error. Because of the communication imperfections from the packet dropout of lossy link, the eventtriggered condition used to determine the data release instant at the event generator (EG) can not be directly applied to update the filter input at the zero order holder $(\mathrm{ZOH})$ when performing filter performance analysis and synthesis. In order to balance such nonuniform time series between the triggered instant of EG and the updated instant of $\mathrm{ZOH}$, two event-triggered conditions are defined, respectively, whereafter a worst-case bound on the number of consecutive packet losses of the transmitted data from EG is given, which marginally guarantees the effectiveness of the filter that will be designed based on the event-triggered updating condition of $\mathrm{ZOH}$. Then, the filter performance analysis conditions are obtained under the assumption that the maximum number of packet losses is allowable for the worst-case bound. In what follows, a two-stage LMI-based alternative optimization approach is proposed to separately design the filter, which reduces the conservatism of the traditional linearization method of filter analysis conditions. Subsequently a codesign algorithm is developed to determine the communication and filter parameters simultaneously. Finally, an illustrative example is provided to verify the validity of the obtained results.
\end{abstract}

\section{Introduction}

Networked control systems (NCSs) use shared communication networks to exchange information among system components such as sensors, controllers, actuators, and filters. These systems have received a persistent attention over the last decade owing to the fact that their architectures guarantee advantages in terms of increased flexibility, reduced wiring and lower maintenance costs, and are finding ways into a wide variety of applications ranging from automation and high-speed signal acquisition systems to process control and power distribution systems. But the introduction of network communication unavoidably results in nonideal quality of service (QoS) because of the imperfections usually characterized as network-induced delay, packet dropout, and protocol constraint [1]. Therefore, the control and estimation problems under such transmission circumstance encounter new challenges and difficulties. In order to handle the network communication imperfection in NCSs, there are several results available in the literature, for instance, the jitter margin approach [2], the frequency-domain approach [3], Lyapunovbased functional approach [4] toward delay and the average system approach [5], worst-case bounds constraint on the number of consecutive dropouts [6], and stochastic modeling approach [7] toward packet dropout. On the basis of the aforementioned techniques to deal with delay and packet loss in NCSs, the networked filtering with imperfect QoS has been considered sufficiently for different systems and various desired performances. Related works can be found in [8-12].

Recently, with the improving attention on resource utilization rates of energy and communication capabilities in NCSs, the event-triggered mechanism is proposed, which 
determines the next transmission time of the needed data based on the previously released data information. The remarkable advantage of this communication strategy mainly lies in the fact that only those signals which are helpful to ensure the desired system performance are transmitted. For example, when the system arrives at the neighborhood of its equilibrium point, this inefficient or redundant system information can be ignored, which saves the network resources to other communication tasks in need on one hand and increases the benefits of energy efficiency on the other hand. In view of these merits of this communication protocol in NCSs, the event-triggered control has been studied extensively. Several representative works can be found in [1320]. In literature, the event-triggered scheme is also referred using various terminologies, such as event-based, eventdriven, dead-band, send-on-delta, and level-crossing [21]. Parallel to the research line of event-triggered control, the networked event-triggered filtering is considered gradually in the past few years. For example, by providing periodic eventtriggered communication scheme, authors in [22] studied a codesign $H_{\infty}$ filtering problem for determined discrete-time systems. Afterwards, through employing information dispatching middleware to establish a framework for networked systems, authors in [23] were concerned with event-triggered $H_{\infty}$ filtering for continuous-time linear systems. In addition, an extended application of event-triggered filtering was given in [24] where the fault detection was performed with communication delay and nonlinear perturbation. Recently, the event-based approach to distributed filtering has been proposed with different triggered manners, such as in [25, 26]. As for the applications of event-triggered method to design filter for the other more general systems, there are also several reported results, for instance, the T-S fuzzy systems [20, 27, 28], the polytopic uncertain systems [29], and the sampled-data systems [30]. However, one can see that the above-mentioned works on event-triggered filtering do not take the packet loss of potentially incomplete communication network into consideration, which may lead to the degradation of system estimation performance when the designed filter is implemented in practice. Therefore, how to design the event-triggered condition under the lossy network communication such that the desired filter can be derived while maintaining the minimized performance evaluation of estimation error is a significant and challenging problem, which is the first motivation of the current study. On the other hand, it should be noted that the existing event-triggered filtering mainly reports on $H_{\infty}$ performance, and to the best of the authors' knowledge, the energy-topeak filtering problem based on event-triggered mechanism has not been stressed. The energy-to-peak theory allows the disturbance signal to be energy bounded and gets the minimum amplitude of the output signal caused by the worstcase disturbance $[31,32]$. Compared with $H_{\infty}$ performance, the energy-to-peak characteristic does not require the output of filtering error system to be square integrable, which has the theoretical and practical significance in some engineering practice, such as the earthquake protection systems of buildings [33]. Therefore, this paper attempts to establish the event-triggered energy-to-peak filter and communication parameters codesign conditions, which is the second motivation of the current research.

Based on the above discussions, this paper studies the event-triggered energy-to-peak filter design problem for a class of discrete-time polytopic uncertain system with the communication consideration of lossy network and quantization. The technical route of this paper can be stated in four steps. Firstly, a time-delay filtering error polytopic system with additive norm-bounded uncertainties is modeled by applying the delay system approach [19] to handle the eventtriggered communication and relying on sector bounded method [34] to deal with the quantization error. Secondly, inspired from the manipulation of packet loss in designing event-triggered controller [17], the estimation of maximum allowable number of consecutive packet losses is calculated, which is used to balance the nonidentical timescales caused by lossy network in event-triggered filter analysis. Thirdly, the energy-to-peak filter analysis and design conditions are deduced under the assumption that the number of packet losses is allowable for the worst-case bound. Fourthly, a codesign algorithm of communication and filter parameters is given, depending on the relationship among the estimation of maximum allowable number of packet losses, the knowledge of the network being used, and the dynamic information of the considered discrete-time system. Finally, the main contributions of this paper are summarized as follows:

(i) The robust energy-to-peak event-triggered filtering problem for polytopic uncertain discrete-time systems with simultaneous consideration of networkinduced delay, signal quantization, and packet loss is studied. Compared with the existing literature on event-triggered filtering, the analysis becomes more complicated because the lossy network leads to the nonuniform triggering time series between the released signal and the successfully received signal. In order to balance such case, the maximum allowable number of consecutive packet dropouts is estimated according to the triggering parameters and the knowledge of the network being used, which is further utilized to enable the general analysis method of event-triggered filtering.

(ii) A two-stage alternative optimization approach to designing energy-to-peak filter is proposed. A twostage optimization approach is proposed to design the energy-to-peak filter. A large conservatism in filter design lies in the information loss during linearization from analysis condition to design condition, which is also the main reason causing optimal solved filtering performance level to deviate from its actual worst-case one largely. Therefore, in order to compensate such information loss, a two-stage optimization algorithm is given to separately design the filter parameters pairs $\left(A_{F}, B_{F}\right)$ and $\left(C_{F}, D_{F}\right)$ on the basis of the linearized design condition with the structural constraints of matrix variables and the directly expanded one, respectively.

(iii) A codesign algorithm is developed to simultaneously determine the desired filter and the threshold 


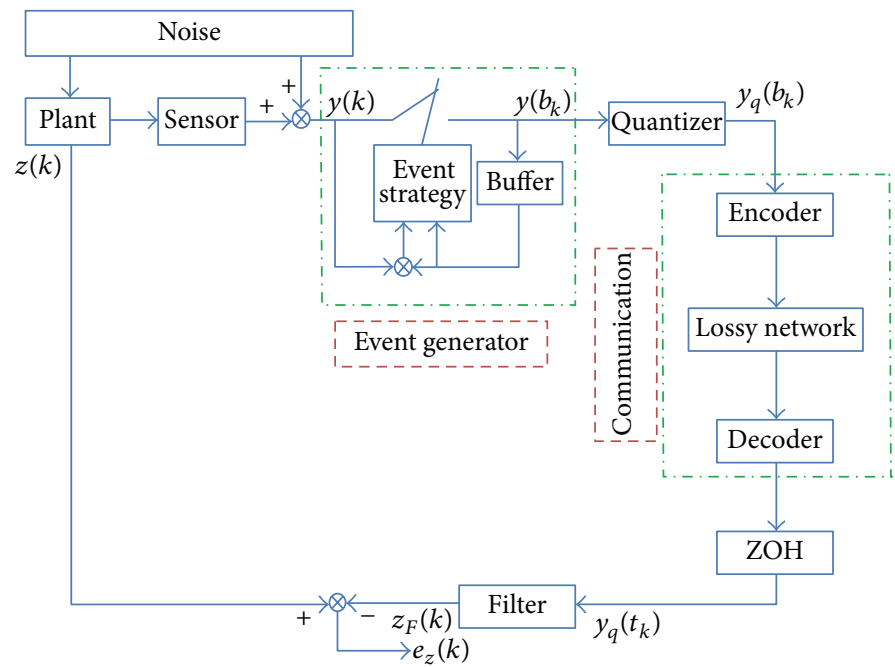

Figure 1: Scheme of event-triggered filtering with lossy network and quantization.

parameters via combining the proposed mathematical relationship of the maximum allowable number of consecutive dropouts and the two-stage alternative optimization method.

The notations are standard. $\mathscr{R}^{n}$ and $\mathscr{R}^{m \times n}$ denote the $n$-dimensional Euclidean space and the set of all $m \times n$ real matrices. The notation $l_{2}[0, \infty)$ represents the space of square-integrable vector functions over $[0, \infty)$. And $H e\{A\}$ and $\operatorname{diag}\{\cdots\}$ denote $A+A^{T}$ and a block-diagonal matrix, respectively. Moreover, $A^{+}$shows the Penrose pseudoinverse of $A$. In addition, $|\cdot|$ stands for the Euclidean vector norm or the induced matrix 2-norm as appropriate. $\lceil\emptyset\rceil$ means the largest integer smaller than or equal to $\emptyset$. Finally, the symbol - represents the symmetric term in a symmetric matrix.

\section{Problem Formulation}

The scheme of event-triggered energy-to-peak filtering for discrete-time polytopic uncertain systems subject to lossy network and quantization error is shown in Figure 1. First, the system output data $y(k)$ is directly transmitted to EG, which is consisted of a buffer and a comparator (event strategy unit). The buffer stores information on the last released data packet and the event strategy unit checks whether the current measured data packet satisfies the triggering condition. Second, the communication capacity of the network is assumed to be limited, and thus the output data $y\left(b_{k}\right)$ of EG is quantized before being transmitted into the network medium for the purpose of reducing the network data transmission rate. Third, due to the collisions, unreliability, or congestion of the network link, the communication channel is not ideal, which is subject to data packet loss. Forth, a logic $\mathrm{ZOH}$ is employed to choose the latest transmitted data packet and discard the disordering packet. Thus, the filter keeps its input signal unchanged until the input of the logic $\mathrm{ZOH}$ gets updated to a new value. Finally, the output of the filter is applied to estimate the concerned output of the plant. In the following, the specified components are described in detail.
2.1. Plant and Energy-to-Peak Filter. Consider the linear time-invariant discrete-time polytopic uncertain system:

$$
\begin{aligned}
x(k+1) & =A(\theta) x(k)+B(\theta) \omega(k), \\
y(k) & =C(\theta) x(k)+D(\theta) \omega(k), \\
z(k) & =L(\theta) x(k)+J(\theta) \omega(k), \\
x(0) & =x_{0},
\end{aligned}
$$

where $x(k) \in \mathscr{R}^{n}$ is the state variable, $\omega(k) \in l_{2}[0, \infty)$ in $\mathscr{R}^{m}$ is the noise signal vector (including process and measurement noises), $z(k) \in \mathscr{R}^{q}$ is the signal to be estimated, and $y(k) \in \mathscr{R}^{f}$ is the measurement output. In addition, the matrices $A(\theta), B(\theta), C(\theta), D(\theta), L(\theta)$, and $J(\theta)$ are system matrices and $C(\theta)$ is full row rank. These system parameters are not precisely known but assumed to reside within the polyhedron:

$$
\begin{aligned}
\Omega & =\{A(\theta), B(\theta), C(\theta), D(\theta), L(\theta), J(\theta)\} \\
& =\sum_{i=1}^{r} \theta_{i}\left\{A_{i}, B_{i}, C_{i}, D_{i}, L_{i}, J_{i}\right\}, \quad \sum_{i=1}^{r} \theta_{i}=1, \theta_{i} \geq 0 .
\end{aligned}
$$

On the other hand, from Figure 1, the designed parameterindependent robust energy-to-peak filter is taken in the form of

$$
\begin{aligned}
x_{F}(k+1) & =A_{F} x_{F}(k)+B_{F} y_{q}\left(t_{k}\right), \\
z_{F}(k) & =C_{F} x_{F}(k)+D_{F} y_{q}\left(t_{k}\right),
\end{aligned}
$$

where $x_{F}(k) \in \mathscr{R}^{n_{F}}$ is the state vector of the full-order filter when $n_{F}=n$ or reduced-order filter when $n_{F}<n$ and $z_{F}(k) \epsilon$ $\mathscr{R}^{q}$ is the filter output. In addition, $y_{q}\left(t_{k}\right)$ characterizes the filter input which is no longer equal to the output of plant due to the communication imperfections and quantization errors. The matrices $A_{F}, B_{F}, C_{F}$, and $D_{F}$ are filter parameters to be determined. 


\subsection{Communication}

2.2.1. Event-Triggered Strategy. The following assumptions are needed to facilitate the theoretical development of eventtriggered scheme $[17,19,20,22,25,35]:$ (1) the sensor is clockdriven while the filter is event-driven; (2) the transmitted signal in network communication is handled by employing the time-stamping technique and it is broadcasted in a singlepacket manner; (3) the total delay $\tau_{k}$ including networkinduced delay from the sensor to the filter and the computational time with waiting delay is bounded; that is, $\tau_{k} \in$ $\left[\tau_{k}^{m}, \tau_{k}^{M}\right]$, where $\tau_{k}^{m}$ and $\tau_{k}^{M}$ denote the lower and upper delay bounds, respectively.

To reduce the event supervision frequency, the discretetime event-triggered mechanism is adopted naturally in this paper. Namely, the EG is running in a periodic manner. Once an event happens, the EG will release the newest system output to the communication channel. In this situation, the following sets are defined:

(1) Let $S_{s}=\left\{0, b_{1}, b_{2}, \ldots, b_{k}, \ldots\right\}$ denote the triggered transmission time sequence at the EG.

(2) Let $S_{r}=\left\{0, t_{1}, t_{2}, \ldots, t_{k}, \ldots\right\}$ represent the successfully received time instant at the $\mathrm{ZOH}$.

Clearly, there is $S_{r} \subseteq S_{s} \subseteq\{0,1, \ldots, k, \ldots\}$. In addition, if all the triggered transmitted outputs at the EG are received successfully at the $\mathrm{ZOH}$, we have $S_{s}=S_{r}$. In the end, the event-triggered condition which determines whether or not the latest sensor measurements will be sent out is given by

$$
\begin{aligned}
& \left(y\left(i_{k}\right)-y\left(b_{k}\right)\right)^{T} \Psi\left(y\left(i_{k}\right)-y\left(b_{k}\right)\right) \\
& \geq \delta_{1} y^{T}\left(b_{k}\right) \Psi y\left(b_{k}\right)
\end{aligned}
$$

where $\Psi>0$ is a weighting matrix and $\delta_{1}>0$ is a threshold. The symbol $i_{k}=b_{k}+i\left(i \in\left\{0,1, \ldots, b_{k+1}-b_{k}\right\}\right)$ is the current measured time instant. Once triggering condition (4) is held, the current measured data packet is immediately released and transmitted to the $\mathrm{ZOH}$ through the communication network. Otherwise, it is discarded off right away. Clearly, the next release time instant $b_{k+1}$ of EG is determined by

$$
\begin{aligned}
b_{k+1}= & b_{k} \\
& +\min _{i \geq 1}\left\{i \mid e^{T}\left(i_{k}\right) \Psi e\left(i_{k}\right) \geq \delta_{1} y^{T}\left(b_{k}\right) \Psi y\left(b_{k}\right)\right\},
\end{aligned}
$$

where $e\left(i_{k}\right)=y\left(i_{k}\right)-y\left(b_{k}\right)$. The parameters $\Psi$ and $\delta_{1}$ denote how frequently the measured output should be transmitted. In general, it is reasonable to set the threshold $\delta_{1}$ on the interval $(0,1)$ from the perspective of relative error because once the value of $\delta_{1}$ is larger than one in the event-triggered condition, the sampled output may be not transmitted after a certain time instant [30]. In addition, the weighting matrix $\Psi$ is introduced to enhance the feasibility of the event-triggered filtering problem.

2.2.2. Quantizer. Besides using triggering techniques to reduce the amount of traffic over network, another way is to quantize the transmitted data. In this paper, the considered quantizers are logarithmic, static, and time invariant which are stated as follows:

$$
\begin{array}{r}
\mathscr{V}=\left\{ \pm \mu_{\ell}: \mu_{\ell}=\rho_{\ell} \mu_{0}, \ell= \pm 1, \pm 2, \ldots\right\} \cup\left\{ \pm \mu_{0}\right\} \cup\{0\} \\
0<\rho_{\ell}<1, \mu_{0}>0,
\end{array}
$$

where the parameter $\rho_{\ell}$ is associated with the quantization density. Obviously, small $\rho_{\ell}$ implies coarse quantization, whereas a large $\rho_{\ell}$ means dense quantization. Each quantization level $\mu_{\ell}$ corresponds to a segment such that the quantizer maps the whole segment to this quantization level. Moreover, these segments form a partition of $\mathscr{R}$. According to the definition of the logarithmic quantizer in [34] and Figure 1, we have

$$
\begin{aligned}
& y_{q}\left(b_{k}\right)=f\left(y\left(b_{k}\right)\right) \\
& = \begin{cases}\rho_{\ell} \mu_{0} & \text { if } \frac{1}{1+\partial_{\ell}} \rho_{\ell} \mu_{0}<y\left(b_{k}\right) \leq \frac{1}{1-\partial_{\ell}} \rho_{\ell} \mu_{0}, \\
0 & \text { if } y\left(b_{k}\right)=0, \\
-f\left(-y\left(b_{k}\right)\right) & \text { if } y\left(b_{k}\right)<0,\end{cases}
\end{aligned}
$$

with

$$
\partial_{\ell}=\frac{1-\rho_{\ell}}{1+\rho_{\ell}} .
$$

It is observed that the above quantizer $f(\cdot)$ is symmetric; that is, $f\left(-y\left(b_{k}\right)\right)=-f\left(y\left(b_{k}\right)\right)$. Defining the measured output quantization error via utilizing the sector bounded method [34], there is

$$
\begin{aligned}
\widetilde{e}\left(b_{k}\right)=y_{q}\left(b_{k}\right)-y\left(b_{k}\right) & =\Delta\left(b_{k}\right) y\left(b_{k}\right), \\
\left|\Delta\left(b_{k}\right)\right| & \leq \partial I, \partial=\operatorname{diag}\left\{\partial_{1}, \partial_{2}, \ldots, \partial_{f}\right\},
\end{aligned}
$$

where $\Delta\left(b_{k}\right)=\operatorname{diag}\left\{\Delta_{1}\left(b_{k}\right), \Delta_{2}\left(b_{k}\right), \ldots, \Delta_{f}\left(b_{k}\right)\right\}$. Then, the quantized output can be given by

$$
y_{q}\left(b_{k}\right)=\left(I+\Delta\left(b_{k}\right)\right) y\left(b_{k}\right) \text {. }
$$

2.2.3. Lossy Network. When performing filtering over networks, packet dropouts can occasionally occur due to data traffic congestion, data collision, or interference [36]. A large number of works in the literature have analysed filtering problem under lossy communication [37, 38]. Generally, there are mainly two different strategies considered for dealing with lossy links: the zero-input and hold-input [39, 40]. In this paper, the hold-input strategy is naturally adopted by virtue of the function of $\mathrm{ZOH}$. Then, parallel to the research ideas in [17], we will handle the influence of lossy network in event-triggered filter design from the perspective of limiting the worst-case bound on the number of consecutive packet dropouts.

2.3. Filtering Error System. Now, we are in a position to model the filtering error system on the basis of the aforementioned parts. First, similar to manipulations of event-triggered mechanism in $[19,41]$ and based on the definitions of delay 
$\tau_{k}$ and sets $S_{s}$ and $S_{r}$ in Section 2.2.1, the holding interval of $\mathrm{ZOH}$ between $\left[t_{k}+\tau_{k}, t_{k+1}+\tau_{k+1}\right)$ can be reconstructed as

$$
\Omega=\left[t_{k}+\tau_{k}, t_{k+1}+\tau_{k+1}\right)=\bigcup_{j=1}^{M_{k}} \Omega_{j},
$$

where $M_{k}=t_{k+1}-t_{k}, \Omega_{j}=\left[\mathrm{o}_{k}-1+\tau_{k}^{j-1}, \mathrm{o}_{k}+\tau_{k}^{j}\right)$, and $\mathrm{a}_{k}=t_{k}+j$, $j=1,2, \ldots, M_{k}-1 . \tau_{k}^{j}$ represents the related delay at the time instant $t_{k}+j$ with $\tau_{k}^{0}=\tau_{k}$ and $\tau_{k}^{t_{k+1}-t_{k}}=\tau_{k+1}$. Moreover, when $j=M_{k}=t_{k+1}-t_{k}, \Omega_{M_{k}}=\left[t_{k+1}-1+\tau_{k}^{M_{k}-1}, t_{k+1}+\tau_{k+1}\right)$. Then, defining $d_{k}=k-a_{k}$ and $e\left(a_{k}\right)=y\left(a_{k}\right)-y\left(t_{k}\right)$, the filter input can be given as

$$
\begin{aligned}
& y_{q}\left(t_{k}\right)=\left(I+\Delta\left(t_{k}\right)\right)\left(y\left(k-d_{k}\right)-e\left(\mathfrak{a}_{k}\right)\right), \\
& \left|\Delta\left(t_{k}\right)\right|<\partial I, k \in \Omega_{j} .
\end{aligned}
$$

In what follows, let $\tilde{x}(k)=\left[x^{T}(k) x_{F}^{T}(k)\right]^{T}, \tilde{\omega}(k)=$ $\left[\omega^{T}(k) \omega^{T}\left(k-d_{k}\right)\right]^{T}$, and $e(k)=z(k)-z_{F}(k)$; for $k \in \Omega_{j}$ we have the augmented filtering error system

$$
\begin{aligned}
\widetilde{x}(k+1)= & \widetilde{A}(\theta) \widetilde{x}(k)+\widetilde{A}_{d}(\theta) H \widetilde{x}\left(k-d_{k}\right) \\
& +\widetilde{B}_{\omega}(\theta) \widetilde{\omega}(k)+\widetilde{B}_{e}(\theta) e\left(\mathfrak{a}_{k}\right), \\
e(k)= & \widetilde{C}(\theta) \widetilde{x}(k)+\widetilde{C}_{d}(\theta) H \widetilde{x}\left(k-d_{k}\right) \\
& +\widetilde{D}_{\omega}(\theta) \widetilde{\omega}(k)+\widetilde{D}_{e}(\theta) e\left({ }^{a_{k}}\right), \\
\widetilde{x}(\kappa)= & {\left[\begin{array}{ll}
x_{0}^{T} & 0
\end{array}\right]^{T}, \quad \kappa \in\left[-\tau_{d}^{M}, 0\right], }
\end{aligned}
$$

where $H=\left[\begin{array}{ll}I & 0\end{array}\right], \widetilde{A}_{d}(\theta)=\widehat{A}_{d}(\theta)+G \Delta\left(t_{k}\right) C(\theta), \widetilde{B}_{\omega}(\theta)=$ $\widehat{B}_{\omega}(\theta)+G \Delta\left(t_{k}\right) S(\theta), \widetilde{B}_{e}=\widehat{B}_{e}-G \Delta\left(t_{k}\right), \widetilde{C}_{d}(\theta)=\widehat{C}_{d}(\theta)-$ $D_{F} \Delta\left(t_{k}\right) C(\theta), \widetilde{D}_{\omega}(\theta)=\widehat{D}_{\omega}(\theta)-D_{F} \Delta\left(t_{k}\right) S(\theta), \widetilde{D}_{e}=\widehat{D}_{e}+$ $D_{F} \Delta\left(t_{k}\right), G=\left[\begin{array}{ll}0 & B_{F}^{T}\end{array}\right]^{T}, S(\theta)=\left[\begin{array}{ll}0 & D \\ & (\theta)\end{array}\right], \widetilde{A}(\theta)=\left[\begin{array}{cc}A(\theta) & 0 \\ 0 & A_{F}\end{array}\right]$, $\widehat{A}_{d}(\theta)=\left[\begin{array}{c}0 \\ B_{F} C(\theta)\end{array}\right], \widehat{B}_{\omega}(\theta)=\left[\begin{array}{cc}B(\theta) & 0 \\ 0 & B_{F} D(\theta)\end{array}\right], \widehat{B}_{e}=\left[\begin{array}{c}0 \\ -B_{F}\end{array}\right], \widetilde{C}(\theta)=$ $\left[L(\theta)-C_{F}\right], \widehat{C}_{d}(\theta)=-D_{F} C(\theta), \widehat{D}_{\omega}(\theta)=\left[J(\theta)-D_{F} D(\theta)\right]$, and $\widehat{D}_{e}=D_{F}$.

Remark 1. Augmented filtering error system (13) is modeled as a system with an interval time-varying delay. Moreover, from the definition of $d_{k}$ above (12), one can obtain $d_{k} \epsilon$ $\left[\tau_{d}^{m}, \tau_{d}^{M}\right]$, where $\tau_{d}^{m}=\min \left\{\tau_{k}^{j-1}-1\right\}, \tau_{d}^{M}=\max \left\{\tau_{k}^{j}\right\}, j=$ $1,2, \ldots, M_{k}$. On the other hand, it should be noted that, due to the fact of the influence introduced by lossy channel link, the filtering error system (13) is updated at the data packet acceptance instant $t_{k}+\tau_{k}$ of $\mathrm{ZOH}$ instead of at the original delayed data release instant $b_{k}+\tau_{k}$ from EG. Hence, compared to (4) it is reasonable to provide a new eventtriggered condition from the filter design point of view. Therefore, assume it in the form of

$$
\begin{aligned}
& \left(y\left(\mathfrak{a}_{k}\right)-y\left(t_{k}\right)\right)^{T} \Psi\left(y\left(\mathfrak{a}_{k}\right)-y\left(t_{k}\right)\right) \\
& \geq \delta_{2} y\left(t_{k}\right)^{T} \Psi y\left(t_{k}\right),
\end{aligned}
$$

where $\delta_{1}<\delta_{2}<1$.
Finally, the objective of this paper can be formulated as follows: under lossy network link, for prescribed positive scalars $\gamma, \tau_{d}^{m}, \tau_{d}^{M}$, determine the event parameters $\delta_{i} \in$ $(0,1), i=1,2$, and $\Psi>0$ and the full-order filter parameters $\left\{A_{F}, B_{F}, C_{F}, D_{F}\right\}$ such that the time-delay dependent filtering error system is asymptotically stable with a given energyto-peak performance level $\gamma$; that is, (1) the filtering error system (13) is asymptotically stable when $\widetilde{\omega}(k)=0 ;(2)$ the filtering error system (13) has a prescribed energy-to-peak disturbance attenuation level $\gamma$ when $\widetilde{\omega}(k) \neq 0$. Namely, under the zero initial condition $\widetilde{x}(0)=0,\|e(k)\|_{\infty}<\gamma\|\widetilde{\omega}(k)\|_{2}$ holds for nonzero $\widetilde{\omega}(k) \in l_{2}[0, \infty)$, where $\|e(k)\|_{\infty}:=$ $\sup _{k}\left\{\sqrt{e^{T}(k) e(k)}\right\},\|\widetilde{\omega}(k)\|_{2}:=\left\{\sqrt{\sum_{k=0}^{\infty} \widetilde{\omega}^{T}(k) \widetilde{\omega}(k)}\right\}$.

The following lemmas are useful to establish our main results.

Lemma 2 (see $[22,42]$ ). For any constant matrices $R \in \mathscr{R}^{n \times n}$ and $U \in \mathscr{R}^{n \times n}, \mathcal{U}=\left[\begin{array}{cc}R & U^{T} \\ U & R\end{array}\right] \geq 0$, scalars $d_{k} \in\left[\tau_{d}^{m}, \tau_{d}^{M}\right]$, and vector function $v:\left[-\tau_{d}^{M},-\tau_{d}^{m}\right] \rightarrow \mathscr{R}^{n}$, such that the following sum is well defined, then

$$
\sum_{j=k-\tau_{d}^{M}}^{k-\tau_{d}^{m}-1} \frac{v^{T}(j) R \nu(j)}{\left(\tau_{d}^{M}-\tau_{d}^{m}\right)^{-1}} \geq\left[\begin{array}{c}
x_{2}-x_{3} \\
x_{3}-x_{4}
\end{array}\right]^{T} \mathcal{U}\left[\begin{array}{l}
x_{2}-x_{3} \\
x_{3}-x_{4}
\end{array}\right],
$$

where $x_{2}=\tilde{x}\left(k-\tau_{d}^{m}\right), x_{3}=\tilde{x}\left(k-d_{k}\right), x_{4}=\tilde{x}\left(k-\tau_{d}^{M}\right)$, and $\nu(k)=\tilde{x}(k+1)-\tilde{x}(k)$.

Lemma 3 (see [43]). For any positive definite matrix $M>0$, two integers $r_{2}$ and $r_{1}$ with $r_{2}>r_{1}$, and a vector valued function $\omega_{i}$, then

$$
\left(\sum_{i=r_{1}}^{r_{2}} \omega_{i}\right)^{T} M\left(\sum_{i=r_{1}}^{r_{2}} \omega_{i}\right) \leq\left(r_{2}-r_{1}+1\right) \sum_{i=r_{1}}^{r_{2}} \omega_{i}^{T} M \omega_{i} .
$$

Lemma 4 (see [44]). Given matrices $\Lambda, \Gamma$ and symmetric matrix $\Omega$, one has that $\Omega+H e\{\Gamma F \Lambda\}<0$ for any $F^{T} F<I$ if and only if there exists a constant scalar $\varepsilon>0$ such that $\Omega+\varepsilon^{-1} \Gamma \Gamma^{T}+\varepsilon \Lambda^{T} \Lambda<0$.

\section{Main Results}

As stated in Remark 1, the lossy network leads to the fact that the event-triggered condition (14) used to formulate the filtering error system is no longer equivalent to the eventtriggered one (4) used to determine the data packet release instant, which further brings the nonuniform time series between EG and $\mathrm{ZOH}$. In order to balance such nonidentical triggering conditions, we first assume that the desired energyto-peak filter can be designed on the basis of (14) with appropriate timing parameters $\delta_{2}$ and $\Psi$. Then, the main work is to find the worst-case bound on the number of consecutive packet dropouts of triggered instants generated by eventtriggered condition (4), which marginally guarantees the effectiveness of the filter designed on the basis of (14).

Theorem 5. The given energy-to-peak disturbance attenuation performance of the filtering error system (13) formulated by (14) 
can be guaranteed under the lossy network, if the number of consecutive dropouts of the released data from the EG with (4), that is, $r_{d p} \in\{0,1, \ldots, l\}$, satisfies

$$
r_{d p} \leq l=\left\lceil\log _{\left(1+\sqrt{\delta_{1}}\right)(1+|\Xi|)} \frac{1+\sqrt{\delta_{2}}}{1+\sqrt{\delta_{1}}}\right\rceil,
$$

where lis the worst-case bound on the number of consecutive packet dropouts.

Proof. Consider the filtering error system (13), and we assume that there are $l$ unsuccessfully transmitted packets in the interval $\left[t_{k}, t_{k+1}\right)$; that is, $t_{k}=b_{p^{0}}<b_{p^{1}}<\cdots<b_{p^{l}}<b_{p^{l+1}}=$ $t_{k+1}$. Then, there is $\left|y\left(b_{p^{l+1}}-1\right)-y\left(b_{p^{l}}\right)\right| \leq \sqrt{\delta_{1}}\left|y\left(b_{p^{l}}\right)\right|$, which further implies $\left|y\left(b_{p^{l+1}}-1\right)\right| \leq\left(1+\sqrt{\delta_{1}}\right)\left|y\left(b_{p^{l}}\right)\right|$. On the other hand, by system output equation, we have $\mid y\left(b_{p^{l+1}}\right)-y\left(b_{p^{l+1}}-\right.$ $1)|=| C(\theta) x\left(b_{p^{l+1}}\right)+D(\theta) \omega\left(b_{p^{l+1}}\right)-y\left(b_{p^{l+1}}-1\right) \mid$, which is equal to $\mid C(\theta) A(\theta) x\left(b_{p^{l+1}}-1\right)+C(\theta) B(\theta) \omega\left(b_{p^{l+1}}-1\right)+D(\theta) \omega\left(b_{p^{l+1}}\right)-$ $y\left(b_{p^{l+1}}-1\right) \mid$. In addition, without loss of generality, we assume that there exists a parameter $\delta_{3}$ such that $|\omega(k)| \leq \delta_{3}|y(k)|$ [17]. Moreover, according to [45], we further assume that the dynamic disturbance can be modeled as follows:

$$
\omega(k+1)=W \omega(k)+\omega(k),
$$

where $W$ is a weighting matrix and $\omega(k)$ is a bounded random function. Then, we have

$$
\begin{aligned}
& \left|y\left(b_{p^{l+1}}\right)-y\left(b_{p^{l+1}}-1\right)\right|=\mid C(\theta) \mathfrak{F}(\theta) y\left(b_{p^{l+1}}-1\right) \\
& \quad+(C(\theta) B(\theta)-C(\theta) \mathfrak{F}(\theta) D(\theta)) \omega\left(b_{p^{l+1}}-1\right) \\
& \quad+D(\theta) \omega\left(b_{p^{l+1}}\right)-y\left(b_{p^{l+1}}-1\right) \mid \\
& \quad \leq\{|C(\theta) \mathfrak{F}(\theta)-I| \\
& \quad+\delta_{3}|C(\theta) B(\theta)-C(\theta) \mathfrak{F}(\theta) D(\theta)| \\
& \left.\quad+\delta_{3} \delta_{4}|D(\theta)|\right\}\left|y\left(b_{p^{l+1}}-1\right)\right| \leq|\Xi|\left|y\left(b_{p^{l+1}}-1\right)\right|,
\end{aligned}
$$

where $\mathfrak{F}(\theta)=A(\theta) C^{+}(\theta),|\Xi|=\max \{|\Xi(\theta)|\}$, and $\delta_{4}=|W|+$ $|\omega|$ that satisfies $|\omega(k+1)| \leq \delta_{4}|\omega(k)|$. Based on the above analysis, it is straightforward to obtain $y\left(b_{p^{l+1}}\right) \leq \mid \Xi \| y\left(b_{p^{l+1}}-\right.$ $1)\left|+\sqrt{\delta_{1}}\right| y\left(b_{p^{l}}\right)|+| y\left(b_{p^{l}}\right)\left|\leq\left(1+\sqrt{\delta_{1}}\right)(1+|\Xi|)\right| y\left(b_{p^{l}}\right) \mid$, which further deduces the following iteration:

$$
\begin{aligned}
y\left(b_{p^{l+1}}\right) & \leq\left(1+\sqrt{\delta_{1}}\right)(1+|\Xi|)\left|y\left(b_{p^{l}}\right)\right| \\
& \leq\left(\left(1+\sqrt{\delta_{1}}\right)(1+|\Xi|)\right)^{2}\left|y\left(b_{p^{l}}-1\right)\right| \leq \cdots \\
& \leq\left(\left(1+\sqrt{\delta_{1}}\right)(1+|\Xi|)\right)^{l+1}\left|y\left(t_{k}\right)\right| .
\end{aligned}
$$

Therefore, for $k \in\left[b_{p^{l}}, b_{p^{l+1}}\right)$, there is

$$
\begin{aligned}
\left|y(k)-y\left(t_{k}\right)\right| \leq & \left|y(k)-y\left(b_{p^{l}}\right)\right| \\
& +\left|\sum_{h=0}^{l-1}\left(y\left(b_{p^{h+1}}-1\right)-y\left(b_{p^{h}}\right)\right)\right| \\
& +\left|\sum_{h=0}^{l-1}\left(y\left(b_{p^{h+1}}\right)-y\left(b_{p^{h+1}}-1\right)\right)\right| \\
\leq & \sum_{h=0}^{l} \sqrt{\delta_{1}}\left|y\left(b_{p^{h}}\right)\right| \\
& +\sum_{h=0}^{l-1}\left(1+\sqrt{\delta_{1}}\right)|\Xi|\left|y\left(b_{p^{h}}\right)\right| .
\end{aligned}
$$

Then relying on (20) to deal with the sums of the geometric progression in (21), we finally obtain

$$
\begin{aligned}
& \left|y(k)-y\left(t_{k}\right)\right| \\
& \quad \leq\left(\left(1+\sqrt{\delta_{1}}\right)^{l+1}(1+|\Xi|)^{l}-1\right) y\left(t_{k}\right) .
\end{aligned}
$$

According to the event-triggered condition (14), we know that inequality (22) holds if the inequality $\left(1+\sqrt{\delta_{1}}\right)^{l+1}(1+|\Xi|)^{l}-$ $1 \leq \sqrt{\delta_{2}}$ holds, which further implies that the performance of the filtering error system formulated by (14) is in accordance with the filtering error system formulated by (4) under lossy network with constraint (17). This completes the proof.

Remark 6. Theorem 5 plays two roles in the subsequent developments of this paper. First, it ensures the effectiveness of utilizing (14) to make filter analysis and design under the actual triggering rule (4) when the communication parameters $\left(\delta_{1}, \delta_{2}, \Psi\right)$ are assumed to be known. Second, it provides the criterion to perform the codesign of communication and filter parameters when the maximum number of consecutive packet dropouts is determined based on the knowledge of the network being used.

Under the constraint of the maximum allowable number of consecutive packet losses estimated in (17), the desired energy-to-peak event-triggered filter can be designed based on (14). In the first place, the performance analysis of eventtriggered filter is given below.

\subsection{Filter Analysis}

Theorem 7. Consider the filtering error system (13) with the communication constraints (14) and (17) for given positive parameters $\tau_{d}^{m}, \tau_{d}^{M}, \delta_{2}, \delta_{1}, \Psi$, and $\gamma$. If there exist matrices $P(\theta)>0, Q_{i}>0, R_{i}>0$, and $\mathfrak{Y}(\theta), U_{j}$ with positive 
scalars $\varepsilon_{1}, \varepsilon_{2}, \vartheta, \chi$ satisfy the following matrix inequalities for $i=1,2,3, j=2,3$, and $\beta=0,1$ :

$$
\begin{aligned}
& {\left[\begin{array}{cccc}
\aleph_{1}(\theta) & \bullet & \bullet & \bullet \\
\mathfrak{V}(\theta) \widehat{\Pi}_{1}(\theta) & P(\theta)-\mathfrak{Y}(\theta)-\mathfrak{V}^{T}(\theta) & \bullet & \bullet \\
0 & (\mathfrak{Y}(\theta) G)^{T} & -\varepsilon_{1} I & \bullet \\
\varepsilon_{1} \partial \Phi(\theta) & 0 & 0 & -\varepsilon_{1} I
\end{array}\right]} \\
& <0 \\
& {\left[\begin{array}{cc}
R_{j} & \bullet \\
U_{j} & R_{j}
\end{array}\right]>0,} \\
& {\left[\begin{array}{cccc}
-\aleph_{2}(\theta) & \bullet & \bullet & \bullet \\
\widehat{\Im}(\theta) & -\gamma^{2} I & \bullet & \bullet \\
0 & D_{F}^{T} & -\varepsilon_{2} I & \bullet \\
\varepsilon_{2} \partial \widehat{\Phi}(\theta) & 0 & 0 & -\varepsilon_{2} I
\end{array}\right]<0,}
\end{aligned}
$$

where

$$
\begin{aligned}
& \aleph_{1}(\theta)=\operatorname{diag}\left\{H^{T}\left(\sum_{i=1}^{3} Q_{i}\right) H-P(\theta), 0,-Q_{1},\right. \\
& \left.-Q_{2},-Q_{3},-I,-\widetilde{\Psi}\right\}+\delta_{2} \Pi_{4}^{T}(\theta) \widetilde{\Psi} \Pi_{4}(\theta)+\beta \Pi_{31} \\
& +(1-\beta) \Pi_{32}+\Theta^{T}(\theta)\left(\sum_{i=1}^{3}\left(d_{i}-d_{i-1}\right)^{2} R_{i}\right) \Theta(\theta), \\
& \widehat{\Pi}_{1}(\theta)=\left[\begin{array}{lllllll}
\widetilde{A}(\theta) & \widehat{A}_{d}(\theta) & 0 & 0 & 0 & \widehat{B}_{\omega}(\theta) & \widehat{B}_{e}
\end{array}\right],
\end{aligned}
$$

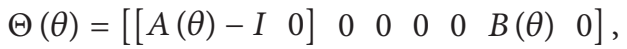

$$
\begin{aligned}
& \Phi(\theta)=\left[\begin{array}{lllllll}
0 & C(\theta) & 0 & 0 & 0 & S(\theta) & -I
\end{array}\right], \\
& \aleph_{2}(\theta)=\operatorname{diag}\left\{P(\theta), Q_{3}, I, \vartheta I\right\},
\end{aligned}
$$

$$
\begin{aligned}
& \widehat{\Im}(\theta)=\left[\begin{array}{llll}
\widetilde{C}(\theta) & \widehat{C}_{d}(\theta) & \widehat{D}_{\omega}(\theta) & \widehat{D}_{e}
\end{array}\right], \\
& \widehat{\Phi}(\theta)=\left[\begin{array}{llll}
0 & -C(\theta) & -S(\theta) & I
\end{array}\right] ;
\end{aligned}
$$

then the filtering error system (13) is asymptotically stable with a guaranteed energy-to-peak performance $\gamma>0$.

Proof. Firstly, a parameter-dependent Lyapunov-Krasovskii function is constructed for (13):

$V(k)$

$$
\begin{aligned}
= & \tilde{x}^{T}(k) P(\theta) \tilde{x}(k)+\sum_{i=1}^{3} \sum_{j=k-d_{i}}^{k-1} \tilde{x}^{T}(j) H^{T} Q_{i} H \tilde{x}(j) \\
& +\sum_{i=1}^{3} \sum_{j=-d_{i}}^{-d_{i-1}-1} \sum_{h=k+j}^{k-1}\left(d_{i}-d_{i-1}\right) \nu^{T}(h) H^{T} R_{i} H \nu(h),
\end{aligned}
$$

where $d_{0}=0, d_{1}=\tau_{d}^{m}, d_{3}=\tau_{d}^{M}, d_{2}=\left\lceil\left(d_{1}+d_{3}\right) / 2\right\rceil$, and $v(h)=\tilde{x}(h+1)-\tilde{x}(h)$. Then, assume any positive integer $N$, nonzero external disturbance, zero initial state condition, and event-triggered manner (14); there is a performance evaluation function for some sufficiently small parameter $\chi>$ 0 :

$$
\begin{aligned}
& \Re(N)=V(N)-\sum_{\eta=0}^{N-1} \widetilde{\omega}^{T}(\eta) \widetilde{\omega}(\eta) \leq \sum_{\eta=0}^{N-1}(\Delta V(\eta) \\
& -\widetilde{\omega}^{T}(\eta) \widetilde{\omega}(\eta) \\
& \left.\quad+\chi\left(\delta_{2} y^{T}\left(t_{k}\right) \Psi y\left(t_{k}\right)-e^{T}\left(\mathfrak{o}_{k}\right) \Psi e\left(a_{k}\right)\right)\right) .
\end{aligned}
$$

Without loss of generality, for any $k \in[0, N-1]$ define $\mathfrak{B}(k)=$ $\underbrace{\Delta V(k)-\tilde{\omega}^{T}(k) \widetilde{\omega}(k)}_{\mathfrak{B}_{1}(k)}+\chi(\underbrace{\delta_{2} y^{T}\left(t_{k}\right) \Psi y\left(t_{k}\right)-e^{T}\left({ }_{a_{k}}\right) \Psi e\left({ }_{k}\right)}_{\mathfrak{B}_{2}(k)})$. Then through direct calculation, we have

$$
\begin{aligned}
\mathfrak{B}_{1}(k)= & \underbrace{\tilde{x}^{T}(k+1) P(\theta) \tilde{x}(k+1)-\tilde{x}^{T}(k) P(\theta) \tilde{x}(k)-\widetilde{\omega}^{T}(k) \widetilde{\omega}(k)}_{\mathfrak{B}_{11}(k)} \\
& +\underbrace{\sum_{i=1}^{3}\left(\tilde{x}^{T}(k) H^{T} Q_{i} H \tilde{x}(k)-\tilde{x}^{T}\left(k-d_{i}\right) H^{T} Q_{i} H \tilde{x}\left(k-d_{i}\right)+\left(d_{i}-d_{i-1}\right)^{2} v^{T}(k) H^{T} R_{i} H \nu(k)\right)}_{\mathfrak{B}_{12}(k)} \\
& -\underbrace{\sum_{i=1}^{3} \sum_{j=k-d_{i}}^{k-d_{i-1}-1}\left(d_{i}-d_{i-1}\right) \nu^{T}(j) H^{T} R_{i} H \nu(j)}_{\mathfrak{B}_{13}(k)}, \\
\mathfrak{B}_{2}(k)= & \delta_{2}\left(\Pi_{4}(\theta) \zeta(k)\right)^{T} \Psi\left(\Pi_{4}(\theta) \zeta(k)\right)-e^{T}\left(\mathfrak{a}_{k}\right) \Psi e\left(a_{k}\right),
\end{aligned}
$$

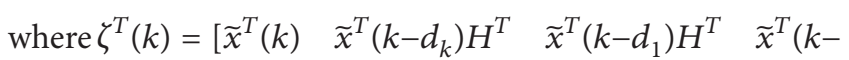
$\left.\left.d_{2}\right) H^{T} \quad \tilde{x}^{T}\left(k-d_{3}\right) H^{T} \quad \tilde{\omega}^{T}(k) \quad e^{T}\left(\mathfrak{d}_{k}\right)\right]$, and $\Pi_{4}(\theta)=$ $\left[\begin{array}{lllllll}0 & C(\theta) & 0 & 0 & 0 & {\left[\begin{array}{ll}0 & D(\theta)\end{array}\right]} & -I\end{array}\right]$. Moreover,

$$
\begin{array}{r}
\mathfrak{B}_{11}(k)=\zeta^{T}(k)\left(\Pi_{1}^{T}(\theta) P(\theta) \Pi_{1}(\theta)\right. \\
-\operatorname{diag}\{P(\theta), 0,0,0,0, I, 0\}) \zeta(k),
\end{array}
$$




$$
\begin{array}{cc}
\mathfrak{B}_{12}(k)=\zeta^{T}(k) & -\left(d_{3}-d_{2}\right) \sum_{i=k-d_{3}}^{k-d_{2}-1} \nu^{T}(i) H^{T} R_{3} H \nu(i) \\
\cdot\left(\Pi_{2}^{T}(\theta) H^{T}\left(\sum_{i=1}^{3}\left(d_{i}-d_{i-1}\right)^{2} R_{i}\right) H \Pi_{2}(\theta)\right. & \leq-\left(H \tilde{x}\left(k-d_{2}\right)-H \tilde{x}\left(k-d_{3}\right)\right)^{T} \\
\left.+\operatorname{diag}\left\{H^{T}\left(\sum_{i=1}^{3} Q_{i}\right) H, 0,-Q_{1},-Q_{2},-Q_{3}, 0,0\right\}\right) & \cdot R_{3}\left(H \tilde{x}\left(k-d_{2}\right)-H \tilde{x}\left(k-d_{3}\right)\right),
\end{array}
$$

$$
\cdot \zeta(k)
$$

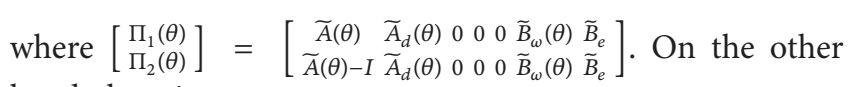
hand, there is

$$
\begin{aligned}
\mathfrak{B}_{13}(k)= & -d_{1} \sum_{i=k-d_{1}}^{k-1} v^{T}(i) H^{T} R_{1} H \nu(i) \\
& -\left(d_{2}-d_{1}\right) \sum_{i=k-d_{2}}^{k-d_{1}-1} v^{T}(i) H^{T} R_{2} H \nu(i) \\
& -\left(d_{3}-d_{2}\right) \sum_{i=k-d_{3}}^{k-d_{2}-1} v^{T}(i) H^{T} R_{3} H \nu(i) .
\end{aligned}
$$

Utilizing the discrete Jensen inequality (Lemma 3) and Lemma 2 to deal with (32), we have

$$
\begin{aligned}
& -d_{1} \sum_{i=k-d_{1}}^{k-1} v^{T}(i) H^{T} R_{1} H \nu(i) \leq-\left(\tilde{x}(k)-\tilde{x}\left(k-d_{1}\right)\right)^{T} \\
& \cdot H^{T} R_{1} H\left(\tilde{x}(k)-\tilde{x}\left(k-d_{1}\right)\right),
\end{aligned}
$$

and if $d_{k} \in\left[d_{1}, d_{2}\right]$, there is

$$
\begin{aligned}
& -\left(d_{2}-d_{1}\right) \sum_{i=k-d_{2}}^{k-d_{1}-1} v^{T}(i) H^{T} R_{2} H v(i) \\
& \leq-\left[\begin{array}{l}
H \tilde{x}\left(k-d_{1}\right)-H \tilde{x}\left(k-d_{k}\right) \\
H \tilde{x}\left(k-d_{k}\right)-H \tilde{x}\left(k-d_{2}\right)
\end{array}\right]^{T} \\
& .\left[\begin{array}{ll}
R_{2} & U_{2}^{T} \\
U_{2} & R_{2}
\end{array}\right]\left[\begin{array}{l}
H \tilde{x}\left(k-d_{1}\right)-H \tilde{x}\left(k-d_{k}\right) \\
H \tilde{x}\left(k-d_{k}\right)-H \tilde{x}\left(k-d_{2}\right)
\end{array}\right],
\end{aligned}
$$

or if $d_{k} \in\left[d_{2}, d_{3}\right]$, there is

$$
\begin{aligned}
& -\left(d_{2}-d_{1}\right) \sum_{i=k-d_{2}}^{k-d_{1}-1} v^{T}(i) H^{T} R_{2} H v(i) \\
& \leq-\left(H \tilde{x}\left(k-d_{1}\right)-H \tilde{x}\left(k-d_{2}\right)\right)^{T} \\
& \cdot R_{2}\left(H \tilde{x}\left(k-d_{1}\right)-H \tilde{x}\left(k-d_{2}\right)\right), \\
& -\left(d_{3}-d_{2}\right) \sum_{i=k-d_{3}}^{k-d_{2}-1} v^{T}(i) H^{T} R_{3} H \nu(i) \\
& \leq-\left[\begin{array}{l}
H \tilde{x}\left(k-d_{2}\right)-H \tilde{x}\left(k-d_{k}\right) \\
H \tilde{x}\left(k-d_{k}\right)-H \tilde{x}\left(k-d_{3}\right)
\end{array}\right]^{T} \\
& .\left[\begin{array}{ll}
R_{3} & U_{3}^{T} \\
U_{3} & R_{3}
\end{array}\right]\left[\begin{array}{l}
H \tilde{x}\left(k-d_{2}\right)-H \tilde{x}\left(k-d_{k}\right) \\
H \tilde{x}\left(k-d_{k}\right)-H \tilde{x}\left(k-d_{3}\right)
\end{array}\right] .
\end{aligned}
$$

Then, from (33)-(35), we get

$$
\begin{array}{ll}
\mathfrak{B}_{13}(k) \leq \zeta^{T}(k) \Pi_{31} \zeta(k), & \text { if } d_{k} \in\left[d_{1}, d_{2}\right], \\
\mathfrak{B}_{13}(k) \leq \zeta^{T}(k) \Pi_{32} \zeta(k), & \text { if } d_{k} \in\left[d_{2}, d_{3}\right],
\end{array}
$$

where

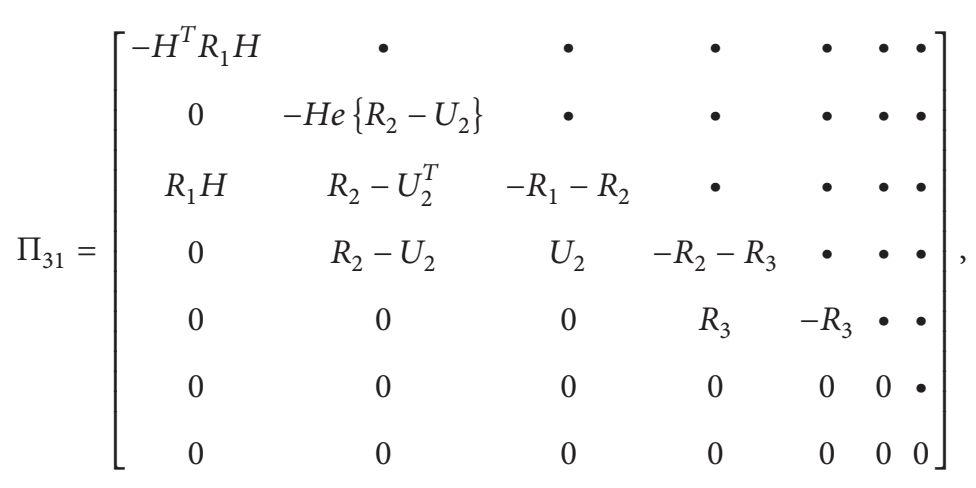




$$
\Pi_{32}=\left[\begin{array}{ccccccc}
-H^{T} R_{1} H & \bullet & \bullet & \bullet & \bullet & \bullet & \bullet \\
0 & -H e\left\{R_{3}-U_{3}\right\} & \bullet & \bullet & \bullet & \bullet & \bullet \\
R_{1} H & 0 & -R_{1}-R_{2} & \bullet & \bullet & \bullet & \bullet \\
0 & R_{3}-U_{3}^{T} & R_{2} & -R_{2}-R_{3} & \bullet & \bullet & \bullet \\
0 & R_{3}-U_{3} & 0 & U_{3} & -R_{3} & \bullet & \bullet \\
0 & 0 & 0 & 0 & 0 & 0 & \bullet \\
0 & 0 & 0 & 0 & 0 & 0 & 0
\end{array}\right] .
$$

At this point, based on (30), (31), and (36), we can conclude that $\mathfrak{B}(k)<0$ if the inequality $\operatorname{diag}\left\{H^{T}\left(\sum_{i=1}^{3} Q_{i}\right) H-P(\theta), 0\right.$, $\left.-Q_{1},-Q_{2},-Q_{3},-I,-\widetilde{\Psi}\right\}+\delta_{2} \Pi_{4}^{T}(\theta) \widetilde{\Psi} \Pi_{4}(\theta)+\beta \Pi_{31}+(1-\beta) \Pi_{32}+$ $\Pi_{2}^{T}(\theta) H^{T}\left(\sum_{i=1}^{3}\left(d_{i}-d_{i-1}\right)^{2} R_{i}\right) H \Pi_{2}(\theta)+\Pi_{1}^{T}(\theta) P(\theta) \Pi_{1}(\theta)<0$ holds, which is equivalent to the following expression:

$$
\left[\begin{array}{cc}
\aleph_{1}(\theta) & \bullet \\
\Pi_{1}(\theta) & -P^{-1}(\theta)
\end{array}\right]<0,
$$

where $\widetilde{\Psi}=\chi \Psi, \beta=0,1$, and $\aleph_{1}(\theta)$ is defined below (25). Then, performing congruence transformation to (38) by $\operatorname{diag}\{I, \mathfrak{V}(\theta)\}$ and applying the elimination lemma (Lemma 4) to deal with the quantization error, (23) can be directly obtained.

Finally, the energy-to-peak filtering performance is established. First, based on $\mathfrak{B}(k)<0$ obtained from (38), there is $\mathfrak{R}(N)<0$ in (28), which further implies $V(k)<$ $\sum_{i=0}^{k-1} \widetilde{\omega}^{T}(i) \widetilde{\omega}(i)$ for any $k$ because of the property of variable irrelevance. In addition, there always exists a sufficiently small parameter $\vartheta>0$ satisfying $V(k)+\vartheta e^{T}\left(\mathfrak{a}_{k}\right) e\left(a_{k}\right) \leq$ $\sum_{i=0}^{k-1} \widetilde{\omega}^{T}(i) \widetilde{\omega}(i)$ due to the properties of the continuity of inequality and S-procedure [46]. On the other hand, inequality (25) implies $e^{T}(k) e(k)<\gamma^{2}\left(\tilde{x}^{T}(k) P(\theta) \tilde{x}(k)+\tilde{x}^{T}(k-\right.$ $\left.d_{k}\right) H^{T} Q_{3} H \tilde{x}\left(k-d_{k}\right)+\widetilde{\omega}^{T}(k) \widetilde{\omega}(k)+\vartheta e^{T}\left(\left(_{k}\right) e\left(\mathbb{d}_{k}\right)\right)$, which further guarantees $e^{T}(k) e(k)<\gamma^{2}\left(V(k)+\widetilde{\omega}^{T}(k) \widetilde{\omega}(k)+\vartheta e^{T}\left(\mathfrak{a}_{k}\right) e\left(\mathfrak{a}_{k}\right)\right)$. Therefore, taking the aforementioned information into consideration, we can conclude $e^{T}(k) e(k)<\gamma^{2}\left(\widetilde{\omega}^{T}(k) \widetilde{\omega}(k)+\right.$ $\left.\sum_{i=0}^{k-1} \widetilde{\omega}^{T}(i) \widetilde{\omega}(i)\right)=\gamma^{2}\left(\sum_{i=0}^{k} \widetilde{\omega}^{T}(i) \widetilde{\omega}(i)\right)$. Finally, taking the supremum of $e^{T}(k) e(k)$ over $k$ and the limit of $\sum_{i=0}^{k} \widetilde{\omega}^{T}(i) \widetilde{\omega}(i)$ with $k \rightarrow \infty$, we have $\|e(k)\|_{\infty}<\gamma\|\widetilde{\omega}(k)\|_{2}$, which completes the proof.

Remark 8. In traditional energy-to-peak performance analysis of filter, it is always assuming zero initial condition, because nonzero initial condition will not lead to the standard "energy ratio" type index. If one makes a point of using nonzero initial condition in filter performance analysis, a corresponding definition of the disturbance attenuation level needs to be modified, such as the similar handling $\|e(k)\|_{\infty}<$ $\gamma\|\widetilde{\omega}(k)\|_{2}+V(0)$ in [47]. Clearly, by doing so, the analysis procedure of Theorem 7 is still available when $\tilde{x}(0) \neq 0$.
Due to the fact that the robustness of the desired eventtriggered filter can be enhanced by minimizing $\gamma$. Namely, the smaller the value of $\gamma$ is, the less the effect from external disturbances on event-triggered filtering error will be. Therefore, we will give the filter design conditions from the perspective of minimizing $\gamma$ in the following part.

3.2. Filter Design. The energy-to-peak filter design conditions can be obtained by linearizing the analysis conditions in Theorem 7. But the traditional linearization methods performed by special structural definition of matrix variable generally lead to the solution information loss of analysis conditions, which finally results in the deviations between the optimized performance of solved event-triggered filter and its actual worst-case level. Therefore, in order to regulate this disequilibrium, we propose the following alternative optimization algorithm in Theorem 9, which makes full use of the combination of the directly expanded matrix variable inequalities of analysis conditions and their traditionally linearized one.

Theorem 9. Let $\tau_{d}^{m}, \tau_{d}^{M}, \delta_{2}, \delta_{1}$, and $\widetilde{\Psi}$ be given, and assume the symmetric matrices $P_{h}>0, Q_{i}>0$, and $R_{i}>0$, matrices $U_{j}$, $A_{F}, B_{F}, \mathscr{A}_{F}, \mathscr{B}_{F}, C_{F}, D_{F}, \mathfrak{V}_{1 h}, \mathfrak{V}_{2 h}, \mathfrak{Y}_{3 h}$, and $\mathfrak{V}_{4 h}$, nonsingular matrix $\mathfrak{V}_{2}$, and scalar parameters $\varepsilon_{1}, \varepsilon_{2}, \vartheta, \alpha_{1}$, and $\alpha_{2}$ be the solutions to the following optimization problems for $(h, s)=$ $1,2, \ldots, r, i=1,2,3, j=2,3$, and $\beta=0,1$ :

(S1)

(1) Solve the following LMI problem:

$$
\begin{aligned}
\gamma_{1}^{*}=\min & \gamma \\
\text { s.t. } & (41),(42),(43)
\end{aligned}
$$

with definitions $\mathfrak{Y}_{2 h}=\alpha_{1} \mathfrak{Y}_{2}, \mathfrak{Y}_{4 h}=\alpha_{2} \mathfrak{Y}_{2}, \mathscr{A}_{F}=$ $\mathfrak{Y}_{2} A_{F}, \mathscr{B}_{F}=\mathfrak{V}_{2} B_{F}$.

(2) Compute $A_{F}=\mathfrak{V}_{2}^{-1} \mathscr{A}_{F}, B_{F}=\mathfrak{V}_{2}^{-1} \mathscr{B}_{F}$.

(1) Use the calculated $A_{F}$ and $B_{F}$ in (S1) to solve the following LMI problem:

$$
\begin{aligned}
\gamma_{2}^{*}=\min & \gamma \\
\text { s.t. } & (41),(42),(43) .
\end{aligned}
$$

(2) Compute $C_{F}$ and $D_{F}$, 
where

$$
\begin{aligned}
& E_{h s}+E_{s h}<0, \quad 1 \leq h \leq s \leq r, \\
& {\left[\begin{array}{cc}
R_{j} & \bullet \\
U_{j} & R_{j}
\end{array}\right]>0} \\
& {\left[\begin{array}{cccc}
-\aleph_{2 h} & \bullet & \bullet & \bullet \\
\widehat{\Im}_{h} & -\gamma^{2} I & \bullet & \bullet \\
0 & D_{F}^{T} & -\varepsilon_{2} I & \bullet \\
\varepsilon_{2} \partial \widehat{\Phi}_{h} & 0 & 0 & -\varepsilon_{2} I
\end{array}\right]<0,} \\
& E_{h s}=\operatorname{diag}\left\{\left[\begin{array}{cc}
\widetilde{\aleph}_{1 h} & \bullet \\
\mathfrak{M}_{h s} & P_{h}-\mathfrak{V}_{h}-\mathfrak{Y}_{h}^{T}
\end{array}\right],\left(\operatorname{diag}\left\{-\left(d_{1}-d_{0}\right)^{-2} R_{1},-\left(d_{2}-d_{1}\right)^{-2} R_{2},-\left(d_{3}-d_{2}\right)^{-2} R_{3},-\widetilde{\Psi},-\varepsilon_{1} I,-\varepsilon_{1} I\right\}\right)\right\} \\
& +\left[\begin{array}{ll}
0 & \bullet \\
\wp_{s} & 0
\end{array}\right]<0 \\
& \widetilde{\aleph}_{1 h}=\left(\operatorname{diag}\left\{H^{T}\left(\sum_{i=1}^{3} Q_{i}\right) H-P_{h}, 0,-Q_{1},-Q_{2},-Q_{3},-I,-\widetilde{\Psi}\right\}\right)+\left(\beta \Pi_{31}+(1-\beta) \Pi_{32}\right), \\
& \widehat{\Pi}_{1 h}=\left[\begin{array}{lllllll}
\widetilde{A}_{h} & \widehat{A}_{d h} & 0 & 0 & 0 & \widehat{B}_{\omega h} & \widehat{B}_{e h}
\end{array}\right] \text {, }
\end{aligned}
$$

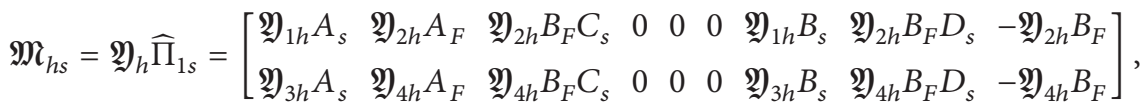

$$
\begin{aligned}
& \wp_{s}^{T}=\left[\begin{array}{cccccc}
\Theta_{s}^{T} R_{1}^{T} & \Theta_{s}^{T} R_{2}^{T} & \Theta_{s}^{T} R_{3}^{T} & \sqrt{\delta_{2}} \widetilde{\Psi} \Pi_{4 s}^{T} & 0 & \varepsilon_{1} \partial \Phi_{s}^{T} \\
0 & 0 & 0 & 0 & {\left[\begin{array}{l}
\mathfrak{Y}_{2 h} B_{F} \\
\mathfrak{Y}_{4 h} B_{F}
\end{array}\right]} & 0
\end{array}\right] \text {, }
\end{aligned}
$$

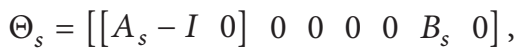

$$
\begin{aligned}
& \Pi_{4 s}=\left[\begin{array}{lllllll}
0 & C_{s} & 0 & 0 & 0 & {\left[\begin{array}{ll}
0 & D_{s}
\end{array}\right]-I}
\end{array}\right] \text {, } \\
& \Phi_{s}=\left[\begin{array}{lllllll}
0 & C_{s} & 0 & 0 & 0 & S_{s} & -I
\end{array}\right], \\
& \aleph_{2 h}=\operatorname{diag}\left\{P_{h}, Q_{3}, I, 9 I\right\}, \\
& \widehat{\Im}_{h}=\left[\begin{array}{llll}
\widetilde{C}_{h} & \widehat{C}_{d h} & \widehat{D}_{\omega h} & \widehat{D}_{e}
\end{array}\right], \\
& \widehat{\Phi}_{h}=\left[\begin{array}{llll}
0 & -C_{h} & -S_{h} & I
\end{array}\right] \text {. }
\end{aligned}
$$

Finally, the event-triggered filter with energy-to-peak performance $\gamma_{2}^{*}$ can be obtained by the combination constituted of $\left(A_{F}, B_{F}\right)$ in $(S 1)$ and $\left(C_{F}, D_{F}\right)$ in (S2).
Proof. First, using Schur's complement to deal with $\aleph_{1}(\theta)$ in (23), we have

$$
\begin{aligned}
& \operatorname{diag}\left\{\left[\begin{array}{lc}
\widetilde{\aleph}_{1}(\theta) & \bullet \\
\mathfrak{M}(\theta) & P_{h}-\mathfrak{Y}(\theta)-\mathfrak{Y}^{T}(\theta)
\end{array}\right],\left(\operatorname{diag}\left\{-\left(d_{1}-d_{0}\right)^{-2} R_{1},-\left(d_{2}-d_{1}\right)^{-2} R_{2},-\left(d_{3}-d_{2}\right)^{-2} R_{3},-\widetilde{\Psi},-\varepsilon_{1} I,-\varepsilon_{1} I\right\}\right)\right\} \\
& +\left[\begin{array}{cc}
0 & \bullet \\
\wp(\theta) & 0
\end{array}\right]<0 .
\end{aligned}
$$


Then, define the structures of matrix variables as

$$
\begin{gathered}
P(\theta)=\sum_{h=1}^{r} \theta_{h}\left[\begin{array}{ll}
P_{1 h} & P_{2 h}^{T} \\
P_{2 h} & P_{3 h}
\end{array}\right], \\
\mathfrak{Y}(\theta)=\sum_{h=1}^{r} \theta_{h}\left[\begin{array}{ll}
\mathfrak{V}_{1 h} & \mathfrak{V}_{2 h} \\
\mathfrak{Y}_{3 h} & \mathfrak{V}_{4 h}
\end{array}\right],
\end{gathered}
$$

and apply them to develop the matrix inequality (45) with the bridging relation; we have

$$
\begin{aligned}
\sum_{h=1}^{r} \sum_{s=1}^{r} \theta_{h} \theta_{s} \mathbb{E}_{h s}= & \sum_{h=1}^{r} \theta_{h}^{2} \mathbb{E}_{h h} \\
& +\sum_{h=1}^{r-1} \sum_{s=h+1}^{r} \theta_{h} \theta_{s}\left(\mathbb{E}_{h s}+\mathbb{E}_{s h}\right)<0,
\end{aligned}
$$

which guarantees the validity of (41). In addition, inequality (43) can be obtained directly from (25) by similar operation.

In the following, we give the two-stage optimization algorithm, respectively. Note that the obstruction preventing us from linearizing inequality (23) is the nonconvex term $\mathfrak{V}_{s} \widehat{\Pi}_{1 h}$. As illustrated before, the traditional linearization will lead to a large conservatism. Hence the work focuses on weakening such drawback. First, define $\mathfrak{Y}_{h}=\left[\begin{array}{lll}\mathfrak{Y}_{1 h} & \alpha_{1} & \mathfrak{V}_{2} \\ \mathfrak{\mathfrak { Y }}_{3 h} & \alpha_{2} & \mathfrak{2} \\ \mathfrak{Y}_{2}\end{array}\right]$, $\mathfrak{2}_{2} A_{F}=\mathscr{A}_{F}$, and $\mathfrak{V}_{2} B_{F}=\mathscr{B}_{F}$; then inequalities (41)-(43) will be LMIs, which further formulate algorithm (S1). Second, preset the obtained value of $A_{F}$ and $B_{F}$ in (S1); inequalities (41)-(43) will be again LMIs when the auxiliary variable $\mathfrak{Y}_{h}$ has the unconstrained structure; that is, $\mathfrak{Y}_{h}=\left[\begin{array}{ll}\mathfrak{Y}_{1 h} & \mathfrak{Y}_{2 h} \\ \mathfrak{Y}_{3 h} & \mathfrak{V}_{4 h}\end{array}\right]$, which verifies algorithm (S2). Third, the two steps (S1) and (S2) can be combined to perform alternative optimization in order to obtain the minimum energy-to-peak performance level. This completes the proof.

Remark 10. The proposed algorithm in Theorem 9 makes an improvement on energy-to-peak filtering performance level. Actually, the first step (S1) is one traditional linearization strategy, which can be also extended to represent the other structural definition methods of matrix variable. But this kind of approach results in information loss of analysis conditions. In order to compensate such defect, algorithm (S2) is given on the basis of the calculated filter dynamic matrices $A_{F}$ and $B_{F}$ in (S1). The intention of enabling (S2) lies in the fact that it can provide the extra solution space relying on the unconstraint matrix variable $\mathfrak{Y}_{h}$. On the other hand, as stated in the above proof, the design conditions in (S1) and (S2) are all LMIbased, which are easy to be solved with SeDuMi [48] and YALMIP [49].

Remark 11. It should be mentioned that according to the required matrix structure of $\mathfrak{Y}_{h}$ in (S1), two scalar parameters $\alpha_{1}$ and $\alpha_{2}$ are added to provide extra freedom of degree. In the literature, there are several ways to find the optimal values of them [50-52]. For simplification, we utilize the tuning scalars algorithm in [52] to solve the optimal event-triggered energyto-peak filtering problem.
3.3. Codesign of Communication and Filter Parameters. In Theorem 9, the event-triggered energy-to-peak filter $\left(A_{F}, B_{F}\right.$, $\left.C_{F}, D_{F}\right)$ is designed under the assumption that the event parameters $\left(\delta_{1}, \delta_{2}, \Psi\right)$ are known. But in the real-world applications these triggering parameters should be determined together with the filter parameters. Hence, a codesign algorithm is indispensable. In addition, the event-triggered filter designed in Theorem 9 is given by way of pursuing the minimum energy-to-peak performance level, which intensifies the objective of this paper. Actually, once given performance value $\gamma$, the desired filter can be designed by (S1) of Theorem 9 in the framework of codesign algorithm. In the following, a codesign algorithm is provided.

Algorithm 12. Find the communication and filter parameters.

(1) Determine $|\Xi|=|\Xi|_{\max }, l$, and $\delta_{3}, \delta_{4}$ based on relationship (19), the knowledge of the network being used, and the dynamic information of the considered system, respectively.

(2) Set initial values $\widetilde{\delta}_{2}=0, \chi=1 e-4$, step increment $H j$, traverse length $H c$, and two related storage variables Hk, Ho.

(3) Let $\widetilde{\delta}_{2}=\widetilde{\delta}_{2}+H j$, and calculate $\widetilde{\delta}_{1}=\left(\left(1+\sqrt{\widetilde{\delta}_{2}}\right) /(1+\right.$ $\left.\left.|\Xi|)^{l}\right)^{1 /(l+1)}-1\right)^{2}$.

(4) If $\widetilde{\delta}_{1}<\widetilde{\delta}_{2}<1$, then $\delta_{2}=\widetilde{\delta}_{2}$ and utilize (S1) of Theorem 9 to calculate $\gamma_{1}^{*}$ and $\Psi$; else if $\widetilde{\delta}_{2}<1$, then go to step (3); else go to step (6).

(5) Store $\gamma_{1}^{*}$ in $H k$ and $\left(\delta_{2}, \Psi\right)$ in $H o$ in a homologous way. And go to step (3) again.

(6) Based on the position of desired $\gamma_{1}^{*}$ in $H k$, find the corresponding $\left(\delta_{2}, \Psi\right)$ in Ho and compute the related $\delta_{1}$ from $\delta_{2}$. Then apply Theorem 9 to calculate the optimized $A_{F}, B_{F}, C_{F}, D_{F}$ with performance $\gamma_{2}^{*}$.

(7) Output $\delta_{1}, \delta_{2}, \Psi, A_{F}, B_{F}, C_{F}, D_{F}, \gamma_{2}^{*}$ and exit.

Remark 13. Several details should be emphasized. First, the parameter $\chi$ is used to ensure the negativeness of $\mathfrak{R}(N)$ in (28) when certain additional terms are introduced. Hence it is reasonable to make it sufficiently small. Second the formula of $\widetilde{\delta}_{1}$ in step (3) is given based on (17) of Theorem 5, which is always satisfying $1>\widetilde{\delta}_{2}>\widetilde{\delta}_{1}>0$. Third, there is no need to apply the two stages of Theorem 9 to calculate $\gamma_{1}^{*}$ and $\Psi$ in step (3) because this step only focuses on finding the suitable $\delta_{2}$. Fourth, in step (6), the parameter $\delta_{2}$ is finally determined from the perspective of minimizing the performance $\gamma$. But one may more prefer to reduce the communication resource occupancy in some practical situation. Therefore, a tradeoff analysis between the communication resource occupancy and the weighted energy-to-peak performance level will be made according to the obtained parameters in $\mathrm{Hk}$ and $\mathrm{Ho}$.

\section{An Illustrative Example}

The LMI problems are solved with SeDuMi [48] and YALMIP [49]. Now, consider a spring-mass system referred from [8, 


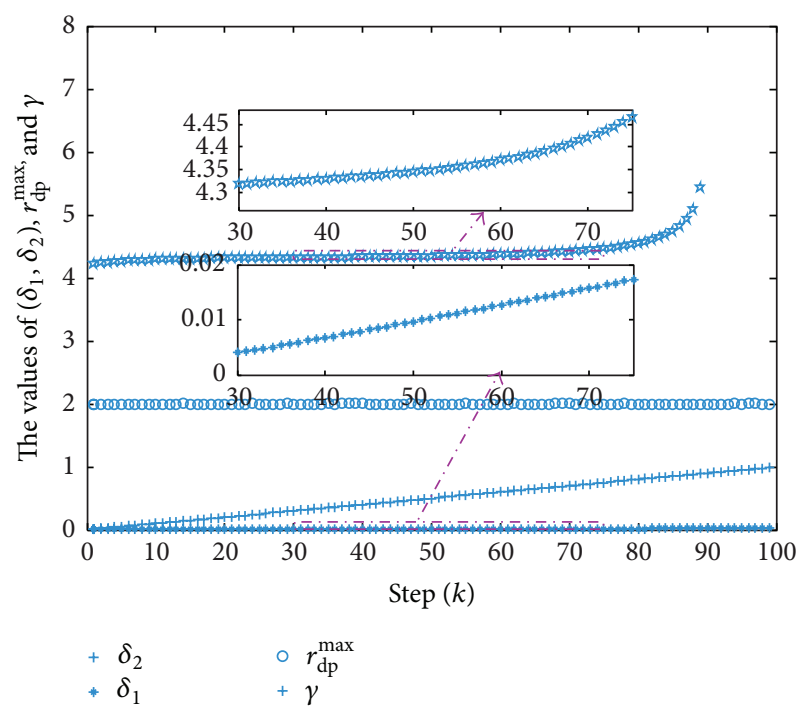

FIGURE 2: The relationships among $\delta_{1}, \delta_{2}, r_{\mathrm{dp}}$, and $\gamma$.

$22,30]$. Assume that the system suffers an uncertain sampling time around $T=0.2 \mathrm{~s}$, which can be described by an uncertain discrete-time model with some parameters inside a polytope [53]. For simplification, we here suppose that the dynamic process of such uncertain model is

$$
\begin{aligned}
x(k+1) & =\left[\begin{array}{cccc}
0.9617+\varrho & 0.0191 & 0.1878 & 0.0012 \\
0.0370 & 0.9629 & 0.0025 & 0.1798 \\
-0.3732 & 0.1853 & 0.8678 & 0.0179 \\
0.3528 & -0.3553 & 0.0357 & 0.7840
\end{array}\right] x(k) \\
& +\left[\begin{array}{c}
0.0193 \\
0.0187 \\
0.1890 \\
0.1813
\end{array}\right] \omega(k),
\end{aligned}
$$

where $|\varrho| \leq 0.2$. Without loss of generality, further let the measurement $y(k)$ and the signal to be estimated $z(k)$ have the matrix coefficients: $C=\left[\begin{array}{llll}1 & 1 & 0 & 0\end{array}\right], D=0.1, L=$ $\left[\begin{array}{llll}1 & 1 & 0 & 0\end{array}\right]$, and $J=0.1$. In this example, set the quantization density to be 0.25 , lower bound of time-varying delay to be $\tau_{d}^{m}=1$, and upper bound of time-varying delay to be $\tau_{d}^{M}=3$, and $\delta_{3}=0.28, \delta_{4}=0.4$, and $H j=0.01$, respectively.

Firstly, we utilize the proposed algorithm in Section 3.3 to show the relationships among event-triggering thresholds $\left(\delta_{1}, \delta_{2}\right)$, the worst-case bound on the number of consecutive packet dropouts $r_{\mathrm{dp}}$, and energy-to-peak performance level $\gamma$, as illustrated in Figure 2. Clearly, in order to weaken the influences of the delay, quantization error, and lossy network, the triggering parameter $\delta_{1}$ stays small. On the other hand, with fixed $\delta_{1}$ and $\delta_{2}$, the maximum allowable number of packet losses is 2 which is calculated by (17). In addition, the different determination of $\delta_{1}$ associated with $\delta_{2}$ has a slender effect on energy-to-peak performance level $\gamma$, which

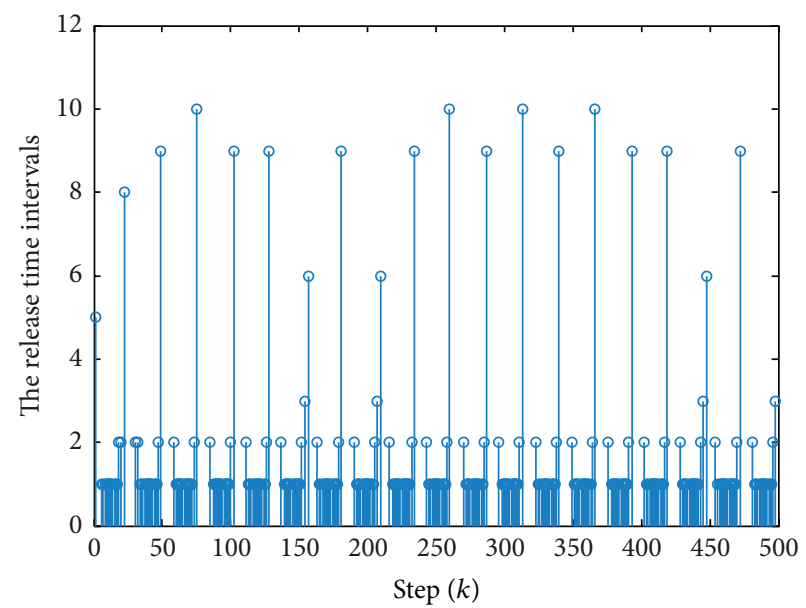

FiguRE 3: The release instants and intervals of event generator.

implies the probability of reducing communication resources occupancy rates while ensuring the desired disturbance attenuation level simultaneously.

Secondly, we verify the less conservatism of Theorem 9. Choose a random triggering threshold $\delta_{1}=0.0173$; then we can obtain the corresponding parameters $\delta_{2}=0.6$ and $\Psi=$ 0.5 from $\mathrm{Ho}$ in codesign algorithm. Next, apply Theorem 9 to calculate the filter parameters; there are

$$
\begin{aligned}
A_{F} & =\left[\begin{array}{cccc}
1.3954 & -0.5083 & 0.2348 & -0.2296 \\
0.2287 & 0.7609 & 0.1259 & -0.0216 \\
-0.4672 & 0.5000 & 0.7762 & 0.2193 \\
0.2737 & -0.5610 & 0.0214 & 0.7886
\end{array}\right], \\
B_{F} & =\left[\begin{array}{c}
0.0575 \\
0.0248 \\
-0.0535 \\
0.0434
\end{array}\right], \\
C_{F} & =\left[\begin{array}{llll}
0.2836 & 0.2687 & 0.1999 & -0.2609
\end{array}\right], \\
D_{F} & =-6.6127 e-15
\end{aligned}
$$

with scalar parameters $\alpha_{1}=-0.2$ and $\alpha_{2}=0.1$ and minimum energy-to-peak performance levels $\gamma_{1}^{*}=4.4671$ and $\gamma_{2}^{*}=$ 4.4566. From the two optimized performance values, we can verify that (S2) further reduces the conservatism of (S1) in Theorem 9.

Thirdly, we show the effectiveness of the designed eventtriggered energy-to-peak filter. Assume the system initial states $x(0)=\left[\begin{array}{llll}-0.01 & -0.02 & 0 & -0.01\end{array}\right]^{T}$ and the external disturbance $\omega(k)=0.02 e^{-0.92 k}$; then the case of the eventtriggered energy-to-peak filtering for the considered polytopic uncertain system with quantization measurement via lossy network is simulated. And the release instants and intervals are depicted in Figure 3, which indicates that eventtriggered scheme reduces the transmitted data number. To illustrate such phenomenon explicitly, the system output 


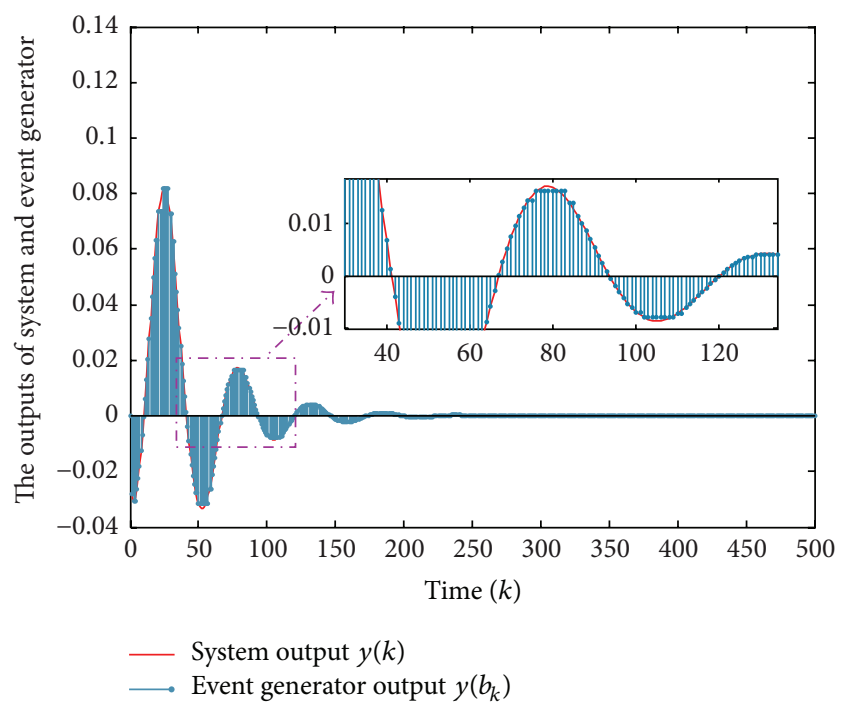

FIGURE 4: The system output signal and actually transmitted signal.

$y(k)$ and the actually triggered output $y\left(b_{k}\right)$ are provided in Figure 4 . And by simple calculation, we obtain that the packet transmission rate under the above event-triggering mechanism is $62 \%$. Hence, the communication resources are saved by $38 \%$. In addition, the random network-induced delay and data packet loss are described in Figure 5. Finally, the estimation error of the preceding solved energy-to-peak filter is shown in Figure 6. Further there are $\|e(k)\|_{\infty}=0.0587$ from Figure 6 and $\|\widetilde{\omega}(k)\|_{2}=0.0235$ by calculation. Therefore, based on the objective given below (14) and the statement in Remark 8, we have the relation $\|e(k)\|_{\infty} /\left(\|\widetilde{\omega}(k)\|_{2}+V(0)\right)<$ $\|e(k)\|_{\infty} /\|\widetilde{\omega}(k)\|_{2}=2.4979<\gamma_{1}^{*}=4.4671$, which shows the effectiveness of the proposed event-triggered energy-to-peak filter design method.

\section{Conclusion}

This paper studies the robust event-triggered energy-to-peak filter design problem for a class of discrete-time polytopic uncertain system with lossy network and quantization error. The packet dropout of lossy network leads to the fact that the transmitted data from the EG may not be received successfully at the $\mathrm{ZOH}$, which further results in the failure of traditional filtering performance analysis method based on event-triggered condition of EG. In order to handle such problem, a boundary condition of consecutive packet dropout from EG is calculated, which ensures that the effectiveness of the filter designed based on received packet at the $\mathrm{ZOH}$ can be still held when the event-triggered communication mechanism of EG is applied in practice. By this strategy, the energy-to-peak filter synthesis conditions are then given via a codesign algorithm associated with a twostage alternative optimization approach. Finally, the validity of the designed filter is verified by an illustrative example.

The calculation method of the worst-case bound constraint on the number of consecutive dropouts is adopted to deal with the lossy network in this paper. Actually, the
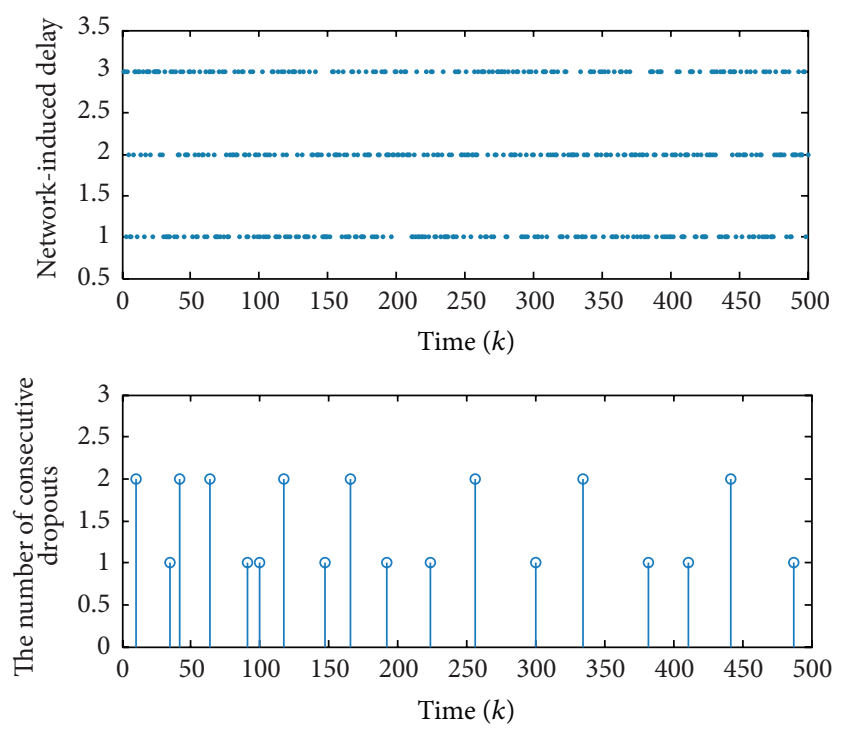

FIgURE 5: The network-induced delay and data packet loss.

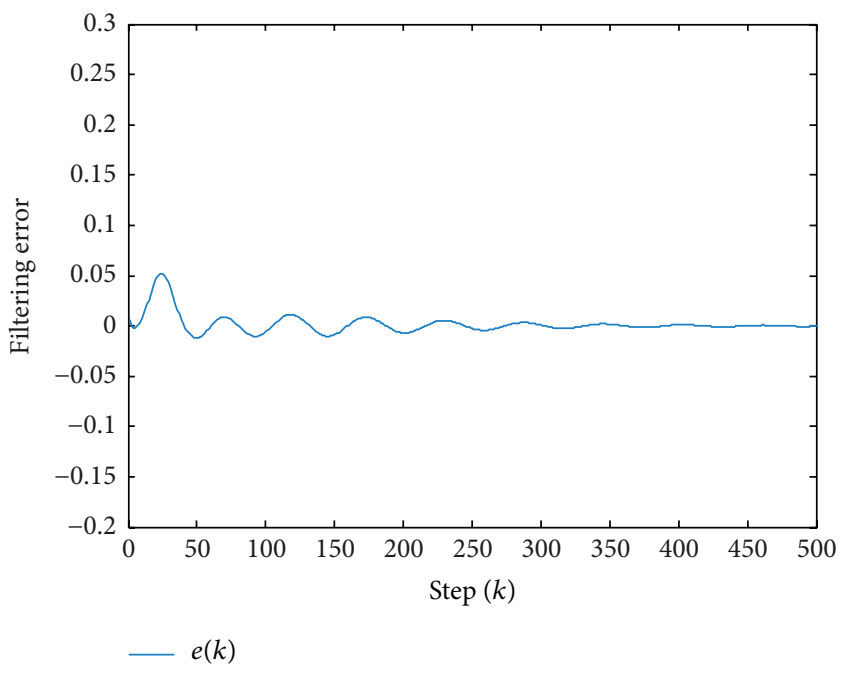

FIGURE 6: The filtering error.

proposed results will be more general if the statistical property of the packet loss is involved. Therefore, how to rely on stochastic modeling approach to handle the above issue needs to be further studied in the future work.

\section{Competing Interests}

The authors declared that they have no competing interests to this work.

\section{Acknowledgments}

This study was supported by the Science and Technology Key Projects of Henan Province (162102210080). 


\section{References}

[1] J. P. Hespanha, P. Naghshtabrizi, and Y. Xu, "A survey of recent results in networked control systems," Proceedings of the IEEE, vol. 95, no. 1, pp. 138-162, 2007.

[2] C.-Y. Kao and B. Lincoln, "Simple stability criteria for systems with time-varying delays," Automatica, vol. 40, no. 8, pp. 14291434, 2004.

[3] C.-Y. Kao and A. Rantzer, "Stability analysis of systems with uncertain time-varying delays," Automatica, vol. 43, no. 6, pp. 959-970, 2007.

[4] H. Gao, T. Chen, and J. Lam, "A new delay system approach to network-based control," Automatica, vol. 44, no. 1, pp. 39-52, 2008.

[5] L. Schenato, B. Sinopoli, M. Franceschetti, K. Poolla, and S. S. Sastry, "Foundations of control and estimation over lossy networks," Proceedings of the IEEE, vol. 95, no. 1, pp. 163-187, 2007.

[6] O. C. Imer, S. Yüksel, and T. Başar, "Optimal control of LTI systems over unreliable communication links," Automatica, vol. 42, no. 9, pp. 1429-1439, 2006.

[7] D. Yue, Q.-L. Han, and C. Peng, "State feedback controller design of networked control systems," IEEE Transactions on Circuits and Systems II: Express Briefs, vol. 51, no. 11, pp. 640644, 2004.

[8] H. Gao and T. Chen, " $H_{\infty}$ estimation for uncertain systems with limited communication capacity," IEEE Transactions on Automatic Control, vol. 52, no. 11, pp. 2070-2084, 2007.

[9] D. Yue and Q.-L. Han, "Network-based robust $H_{\infty}$ filtering for uncertain linear systems," IEEE Transactions on Signal Processing, vol. 54, no. 11, pp. 4293-4301, 2006.

[10] B. Jiang, Z. Mao, and P. Shi, " $H_{\infty}$-filter design for a class of networked control systems via T-S fuzzy-model approach," IEEE Transactions on Fuzzy Systems, vol. 18, no. 1, pp. 201-208, 2010.

[11] H. Dong, Z. Wang, J. Lam, and H. Gao, "Distributed filtering in sensor networks with randomly occurring saturations and successive packet dropouts," International Journal of Robust and Nonlinear Control, vol. 24, no. 12, pp. 1743-1759, 2014.

[12] H. Zhang, Y. Shi, and A. S. Mehr, "Robust energy-to-peak filtering for networked systems with time-varying delays and randomly missing data," IET Control Theory and Applications, vol. 4, no. 12, pp. 2921-2936, 2010.

[13] D. Lehmann and J. Lunze, "Event-based control with communication delays and packet losses," International Journal of Control, vol. 85, no. 5, pp. 563-577, 2012.

[14] M. Guinaldo, D. Lehmann, J. Sanchez, S. Dormido, and K. H. Johansson, "Distributed event-triggered control for nonreliable networks," Journal of the Franklin Institute, vol. 351, no. 12, pp. 5250-5273, 2014.

[15] F.-L. Qu, Z.-H. Guan, D.-X. He, and M. Chi, "Event-triggered control for networked control systems with quantization and packet losses," Journal of the Franklin Institute, vol. 352, no. 3, pp. 974-986, 2015.

[16] J. Zhang and G. Feng, "Event-driven observer-based output feedback control for linear systems," Automatica, vol. 50, no. 7, pp. 1852-1859, 2014.

[17] C. Peng and T. C. Yang, "Event-triggered communication and $H_{\infty}$ control codesign for networked control systems," Automatica, vol. 49, no. 5, pp. 1326-1332, 2013.
[18] X. H. Chang, H. Ju, and J. Zhou, "Robust static output feedback $H_{\infty}$ control design for linear systems with polytopic uncertainties," Systems \& Control Letters, vol. 85, pp. 23-32, 2015.

[19] D. Yue, E. Tian, and Q.-L. Han, "A delay system method for designing event-triggered controllers of networked control systems," IEEE Transactions on Automatic Control, vol. 58, no. 2, pp. 475-481, 2013.

[20] X.-C. Jia, X.-B. Chi, Q.-L. Han, and N.-N. Zheng, "Eventtriggered fuzzy $H_{\infty}$ control for a class of nonlinear networked control systems using the deviation bounds of asynchronous normalized membership functions," Information Sciences, vol. 259, pp. 100-117, 2014.

[21] M. S. Mahmoud and A. M. Memon, "Aperiodic triggering mechanisms for networked control systems," Information Sciences, vol. 296, pp. 282-306, 2015.

[22] C. Peng and M.-R. Fei, "Networked $H_{\infty}$ filtering for discrete linear systems with a periodic event-triggering communication scheme," IET Signal Processing, vol. 7, no. 8, pp. 754-765, 2013.

[23] Y. Lin, Q. L. Han, F. Yang, and D. Jarvis, "Event-triggered $H_{\infty}$ filtering for networked systems based on network dynamics," in Proceedings of the 39th Annual Conference of the IEEE Industrial Electronics Society (IECON '13), pp. 5638-5643, Vienna, Austria, November 2013.

[24] J. Liu and D. Yue, "Event-based fault detection for networked systems with communication delay and nonlinear perturbation," Journal of the Franklin Institute, vol. 350, no. 9, pp. 27912807, 2013.

[25] X. Ge and Q.-L. Han, "Distributed event-triggered $H_{\infty}$ filtering over sensor networks with communication delays," Information Sciences, vol. 291, pp. 128-142, 2015.

[26] L. Yan, X. Zhang, Z. Zhang, and Y. Yang, "Distributed state estimation in sensor networks with event-triggered communication," Nonlinear Dynamics, vol. 76, no. 1, pp. 169-181, 2014.

[27] J. Liu, S. Fei, E. Tian, and Z. Gu, "Co-design of event generator and filtering for a class of T-S fuzzy systems with stochastic sensor faults," Fuzzy Sets and Systems, vol. 273, pp. 124-140, 2015.

[28] H. Wang, P. Shi, and J. Zhang, "Event-triggered fuzzy filtering for a class of nonlinear networked control systems," Signal Processing, vol. 113, pp. 159-168, 2015.

[29] X. Meng and T. Chen, "Event triggered robust filter design for discrete-time systems," IET Control Theory \& Applications, vol. 8, no. 2, pp. 104-113, 2014.

[30] X.-M. Zhang and Q.-L. Han, "Event-based $H_{\infty}$ filtering for sampled-data systems," Automatica, vol. 51, pp. 55-69, 2015.

[31] L. Wu and D. W. C. Ho, "Reduced-order $l_{2}-l_{\infty}$ filtering for a class of nonlinear switched stochastic systems," IET Control Theory and Applications, vol. 3, no. 5, pp. 493-508, 2009.

[32] J. Qiu, G. Feng, and J. Yang, "New results on robust energyto-peak filtering for discrete-time switched polytopic linear systems with time-varying delay," IET Control Theory and Applications, vol. 2, no. 9, pp. 795-806, 2008.

[33] W. Zhang, Y. Chen, and H. Gao, "Energy-to-peak control for seismic-excited buildings with actuator faults and parameter uncertainties," Journal of Sound and Vibration, vol. 330, no. 4, pp. 581-602, 2011.

[34] M. Fu and L. Xie, "The sector bound approach to quantized feedback control," IEEE Transactions on Automatic Control, vol. 50, no. 11, pp. 1698-1711, 2005.

[35] S. Hu and D. Yue, "Event-triggered control design of linear networked systems with quantizations," ISA Transactions, vol. 51, no. 1, pp. 153-162, 2012. 
[36] B. Demirel, V. Gupta, and M. Johansson, "On the trade-off between control performance and communication cost for event-triggered control over lossy networks," in Proceedings of the 12th European Control Conference (ECC '13), pp. 1168-1174, IEEE, Zurich, Switzerland, July 2013.

[37] B. Sinopoli, L. Schenato, M. Franceschetti, K. Poolla, M. I. Jordan, and S. S. Sastry, "Kalman filtering with intermittent observations," IEEE Transactions on Automatic Control, vol. 49, no. 9, pp. 1453-1464, 2004.

[38] L. Schenato, "Optimal estimation in networked control systems subject to random delay and packet drop," IEEE Transactions on Automatic Control, vol. 53, no. 5, pp. 1311-1317, 2008.

[39] L. Schenato, "To zero or to hold control inputs with lossy links?" IEEE Transactions on Automatic Control, vol. 54, no. 5, pp. $1093-$ 1099, 2009.

[40] A. R. Fioravanti, A. P. C. Goncalves, and J. C. Geromel, "Filter inputs with markovian lossy links: zero or hold?" in Proceedings of the 9th International Conference on Control and Automation (ICCA '11), pp. 656-661, IEEE, Santiago, Chile, December 2011.

[41] X. M. Zhang and Q. L. Han, "Event-based dynamic output feedback control for networked control systems," in Proceedings of the American Control Conference (ACC '13), pp. 3008-3013, IEEE, Washington, DC, USA, June 2013.

[42] C. Peng and M.-R. Fei, "An improved result on the stability of uncertain T-S fuzzy systems with interval time-varying delay," Fuzzy Sets and Systems, vol. 212, pp. 97-109, 2013.

[43] K. Gu, V. L. Kharitonov, and J. Chen, Stability of Time-delay Systems, Birkhäauser, Boston, Mass, USA, 2003.

[44] I. R. Petersen, "A stabilization algorithm for a class of uncertain linear systems," Systems \& Control Letters, vol. 8, no. 4, pp. 351357, 1987.

[45] F. Farokhi, C. Langbort, and K. H. Johansson, "Decentralized disturbance accommodation with limited plant model information," SIAM Journal on Control and Optimization, vol. 51, no. 2, pp. 1543-1573, 2013.

[46] H. Liu, D. W. C. Ho, and F. Sun, "Design of $H_{\infty}$ filter for Markov jumping linear systems with non-accessible mode information," Automatica, vol. 44, no. 10, pp. 2655-2660, 2008.

[47] Y. K. Foo, " $H_{\infty}$ fault detection with randomly occurring nonlinearities and channel fadings," IEEE Transactions on Circuit System-II Express Briefs, vol. 53, no. 11, pp. 1220-1224, 2006.

[48] J. F. Sturm, "Using SeDuMi 1.02, a MATLAB toolbox for optimization over symmetric cones," Optimization Methods and Software, vol. 11-12, no. 1-4, pp. 625-653, 1999.

[49] J. Löfberg, "YALMIP: a toolbox for modeling and optimization in MATLAB," in Proceedings of the IEEE International Symposium on Computer Aided Control System Design, pp. 284-289, IEEE, Taipei, Taiwan, September 2004.

[50] C. F. Morais, M. F. Braga, R. C. L. F. Oliveira, and P. L. D. Peres, "Robust state feedback control for discrete-time linear systems via LMIs with a scalar parameter," in Proceedings of the American Control Conference (ACC '13), pp. 3870-3875, IEEE, Washington, DC, USA, June 2013.

[51] L. Xie, L. Lu, D. Zhang, and H. Zhang, "Improved robust $H_{2}$ and $H_{\infty}$ filtering for uncertain discrete-time systems," Automatica, vol. 40, no. 5, pp. 873-880, 2004.

[52] K. Han and J. Feng, "Improved scalar parameters approach to design robust $H_{\infty}$ filter for uncertain discrete-time linear systems," Signal Processing, vol. 113, pp. 113-123, 2015.
[53] R. A. Borges, R. C. L. F. Oliveira, C. T. Abdallah, and P. L. D. Peres, "Robust $H_{\infty}$ networked control for systems with uncertain sampling rates," IET Control Theory and Applications, vol. 4, no. 1, pp. 50-60, 2010. 


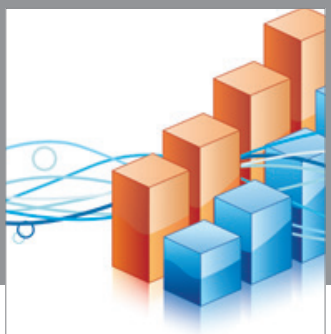

Advances in

Operations Research

vatem alat4

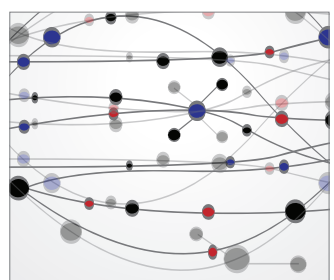

\section{The Scientific} World Journal
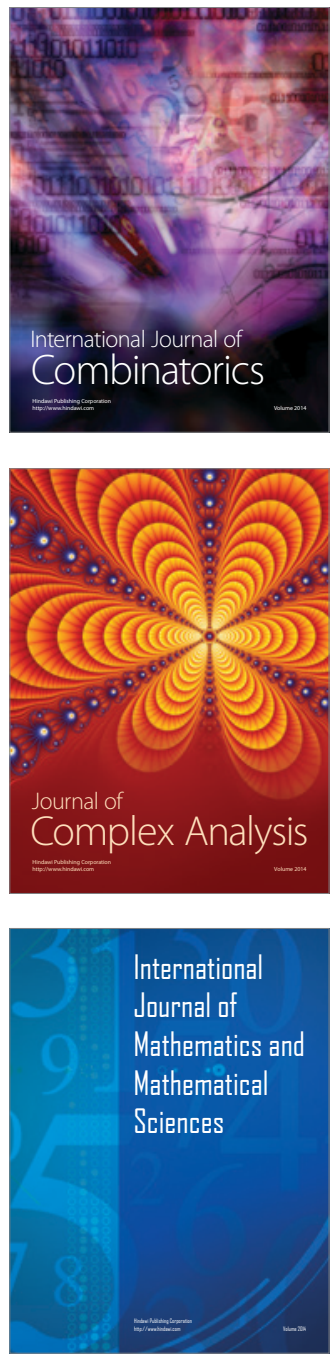
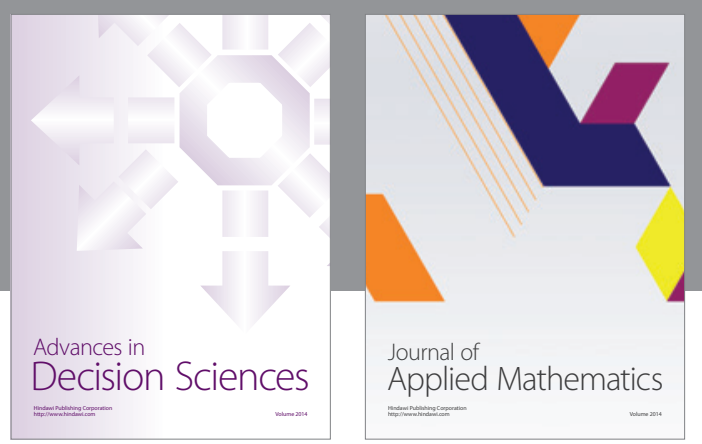

Algebra

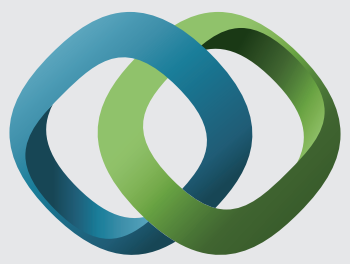

\section{Hindawi}

Submit your manuscripts at

http://www.hindawi.com
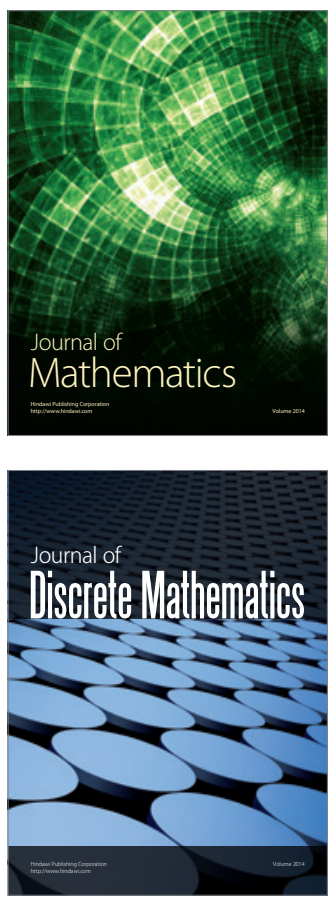

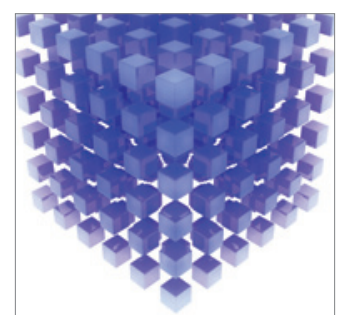

Mathematical Problems in Engineering
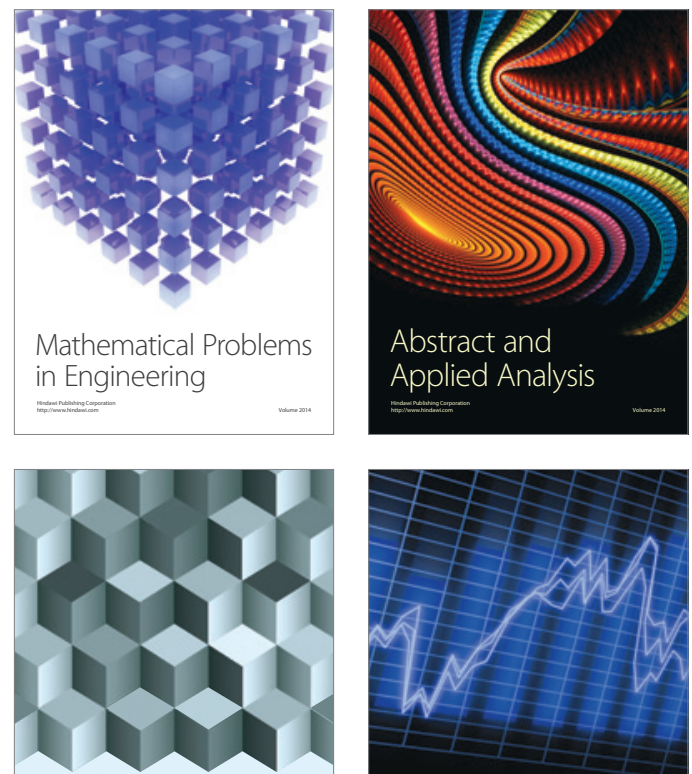

Journal of

Function Spaces

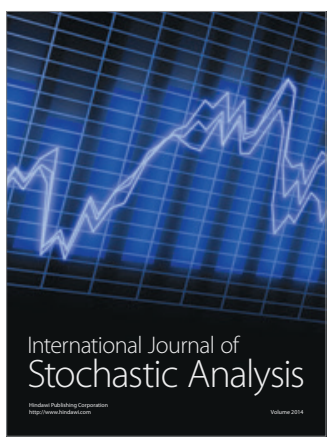

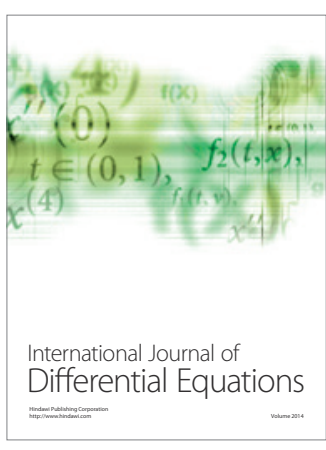
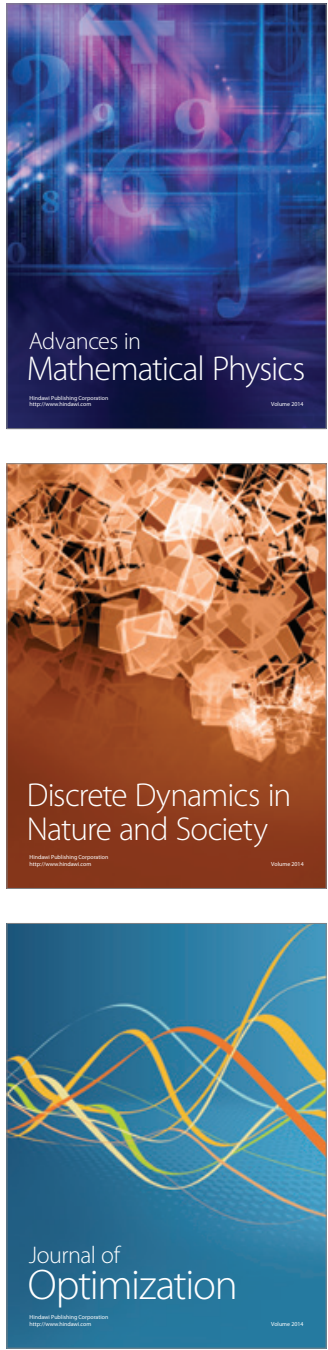